\title{
ASYMPTOTIC COMPLETENESS OF $N$-PARTICLE LONG-RANGE SCATTERING
}

\author{
I. M. SIGAL AND A. SOFFER
}

\section{INTRODUCTION}

In this paper we prove asymptotic completeness for $N$-particle long-range systems with potentials vanishing at infinity as $O\left(|x|^{-\mu}\right)$ with $\mu \geq 1-2^{-N-2}$. Previously asymptotic completeness for long-range quantum systems for two particles and various classes of potentials was proven by several authors (see [Sig2] for references) and for three particles with pair interactions vanishing at $\infty$ as $O\left(|x|^{-\mu}\right.$ ) with $\mu>\sqrt{3}-1$ in [En2] (see also [SigSof5] for a different proof and see [Mo] for another approach). Asymptotic completeness for $N$ particle Schrödinger operators with two generations of thresholds (this, in particular, includes general four particle operators) and interacting via pair potentials vanishing at $\infty$ as $O\left(|x|^{-1}\right.$ ) (Coulomb-type potentials) was proven in [SigSof6].

Our approach consists of two steps. In the first step we use asymptotic clustering to reduce the problem involving an $(N+1)$-particle Schrödinger operator to that involving a time-dependent operator of the form $H(t)=H+W(x, t)$ where $H$ is an $N$-particle Schrödinger operator and $W(x, t)$ is a real potential obeying $\left|\partial^{\alpha} W(x, t)\right| \leq C_{\alpha}(1+|x|+|t|)^{-\mu-|\alpha|}$. The asymptotic clustering states roughly that as $t \rightarrow \pm \infty$ a system in question disintegrates into noninteracting, and therefore freely moving, subsystems. In the short-range case $(\mu>1), W$ is in $L^{1}(d t)$ and therefore can be dropped. Using this one shows that asymptotic clustering for nonthreshold energies implies asymptotic completeness. In the long-range case $W(x, t)$ cannot be dropped. Since the energy is not conserved for $H(t)$, dealing with it poses a number of subtle problems. $H(t)$ is handled in the second step. In this step we use a fine microlocal analysis on ranges of singular asymptotic projections in order to reduce the problem of controlling the evolution $U(t)$ generated by $H(t)$ to the one of controlling the evolution generated by $H$.

To explain this approach in more precise terms we introduce some notation. For a family $B=\left\{B_{t}, t>0\right\}$ of selfadjoint operators and a Borel set $\Delta$ with

Received by the editors April 6, 1993.

1991 Mathematics Subject Classification. Primary 81U10, 35P25, 47F05.

The first author was supported by NSERC under Grant \# NA7901 and I. W. Killam Research Fellowship, and the second author was supported by NSF under Grant DMS85-07040. 
$|\Delta|>0$, we define

$$
F_{\Delta}^{+}(B)=\mathrm{s}-\lim _{t \rightarrow+\infty} U(t)^{*} F_{\Delta}\left(B_{t}\right) U(t)
$$

where $F_{\Delta}(\lambda)$ is a smoothed-out characteristic function of $\Delta$ (see the end of this introduction). For $|\Delta|=0$ we define $F_{\Delta}^{+}(B)$ by a limiting procedure (see $\S 4$ ). Denote $v=\left\{\frac{|x|}{t}\right\}$. Let $\tau$ be the threshold set of $H . \tau$ and $\{0\}$ are the singular, from the point of view of propagation, sets for $H$ and $\frac{|x|}{t}$, respectively, and $\operatorname{Ran} F_{\{0\}}^{+}(v) \subset \operatorname{Ran} F_{\tau}^{+}(H)$. We show that $U(t)$ is asymptotically clustering on $\left(\mathscr{R}_{0}^{+}\right)^{\perp}$, where $\mathscr{R}_{0}^{+}=\operatorname{Ran} F_{\{0\}}^{+}(v)$, i.e., it is reduced to the evolutions generated by (time-dependent) Hamiltonians of noninteracting subsystems. This yields the reduction in the number of particles. Furthermore, we show that on $\mathscr{R}_{0}^{+}, U(t)$, modulo uniformly in $t$ small and asymptotically clustering terms, concentrates in the set $\left\{|x| \leq t^{\alpha}\right\}$ with $\alpha<\mu$. This allows one to pass from $U(t)$ to the evolution $U_{0}(t)$ generated by $H_{0}(t)=H+W(0, t)$, i.e., one is back to a time-independent Hamiltonian. The case $t \rightarrow-\infty$ is treated similarly.

To sum up, in the second step one characterizes subspaces of balistic and subbalistic propagation in terms of singular sets for (or the spectra of) appropriate asymptotic observables.

The method described above is a close extension of the method of [SigSof6]. Asymptotic cut-offs $F_{\Delta}^{+}(H)$ were introduced in [SigSof5], while cut-offs related to $F_{\Delta}^{+}(v)$, in [Gr]. In the present context $F_{\Delta}^{+}(v)$ can be replaced by asymptotic projections $F_{\Delta}^{+}(K)$ for $K=\left\{\frac{A}{t}\right\}$ (see, e.g., $\S 7$ and supplement of [SigSof6]). In fact, $F_{\{0\}}^{+}(v)=F_{\{0\}}^{+}(K) . F_{\Delta}^{+}(K)$ were used implicitly in [SigSof6] (see Introduction and $\S 5$, and especially Eqs. (5.10) and (5.13)). Asymptotic observables in the context of the QM scattering theory were first used in [En1]. For recent important results see [Der2, En3]. Our treatment of asymptotic clustering follows [SigSof6, GerDer], which extend earlier results of [SigSof3]. A beautiful proof of key estimates of [SigSof3] was given in [Gr]. Important ideas introduced in this proof are used in the present paper (see Appendix). Our estimates on $\mathscr{R}_{0}^{+}$are essentially a simplified version of estimates of [SigSof6]. The latter paper proves the asymptotic clustering on $\left(\mathscr{R}_{\tau, \varepsilon}^{+}\right)^{\perp} \forall \varepsilon>0$ and the reduction to a time-independent Schrödinger operator, on $\mathscr{R}_{\tau, \varepsilon}^{+}$, for $\varepsilon>0$ sufficiently small. Here $\mathscr{R}_{\Delta, \varepsilon}^{+}=\operatorname{Ran} F_{\Delta}^{+}(H) \cap \operatorname{Ran} F_{[-\varepsilon, \varepsilon]}^{+}(K)$. An important step of taking $\varepsilon=0$, i.e., restricting not only to the singular subset $\tau$ of $H$, but also to the singular subset $\{0\}$ of $\frac{A}{t}$ (or of $\frac{|x|}{t}$ ), and proving the sharp localization of $x$ on this subspace directly (rather than using the sharp localization of $H$ as was done in [SigSof6]), was suggested by [Der3]. These ideas inspired the result of Theorem 5.1, extending Theorem 13.2 of [SigSof6]. This theorem is an important ingredient in our microlocal analysis of $U(t) \psi$ with $\psi \in \mathscr{R}_{0}^{+}\left(=\mathscr{R}_{\varepsilon, 0}^{+}\right)$.

In this paper we use the following convention for cut-off functions. In general, $F(\lambda \in \Omega)$, for a Borel set $\Omega \subset \mathbb{R}$, stands for a smoothed out characteristic function of $\Omega$. More precisely, let $\omega$ be a smooth function on $\mathbb{R}$, supported in $|\lambda| \leq 2$ and satisfying $\omega \geq 0, \int \omega=1$, and $\omega(0)=1$. Let $\omega_{\delta}(\lambda)=\delta^{-1} \omega\left(\frac{\lambda}{\delta}\right)$. 
If $|\Omega|>0$, then we set

$$
F(\lambda \in \Omega)=\chi_{\Omega} * \omega_{\delta},
$$

where $\chi_{\Omega}$ is the characteristic function of $\Omega$ and $\delta \leq|\Omega|$. Thus we have

$$
F(\lambda \in \Omega)+F(\lambda \in(\mathbb{R} \backslash \Omega))=1 \text {. }
$$

Moreover, we set for any $a \in \mathbb{R}$

$$
F(\lambda=a)=\delta F^{\prime}(\lambda \geq a)=\omega\left(\frac{\lambda-a}{\delta}\right) .
$$

The parameter $\delta^{-1}$ will be called the sharpness of the cut-off function in question. Let $|\Omega|$ denote the Lebesgue measure of $\Omega \subset \mathbb{R}$. Then for $F\left(\lambda \geq \lambda_{0}\right)$ the sharpness is reciprocal to $\delta=\left|\operatorname{supp} F^{\prime}\left(\lambda \geq \lambda_{0}\right)\right|$ and similarly for $F\left(\lambda \leq \lambda_{0}\right)$. Symbol $\langle x\rangle$ stands for a smooth function equal to $|x|$ for $|x| \geq 1$ and not less than $\frac{1}{2}$ for $|x| \leq 1$. Note that $\langle x\rangle$ is positive homogeneous of degree 1 for $|x| \geq 1$ and is invertible. The main statements are formulated for $t \rightarrow \pm \infty$, while all the other statements as well as the proofs are given for $t \rightarrow \infty$ only. $O_{i}\left(R^{-1}\right)$, where $R$ is either $\langle x\rangle^{\alpha}$ or $\langle t\rangle^{\alpha}$, stands for an operator s.t. for every $s \in[0,1]$ and for every $k=0, \ldots, i, R^{s}(-\Delta+1)^{-k} O_{i}\left(R^{-1}\right)(-\Delta+1)^{-i+k} R^{1-s}$ extends to a bounded operator.

\section{HAMILTONIANS AND KINEMATICS}

Consider an $N$-body system in the physical space $R^{\nu}$. The configuration space in the center-of-mass frame is ([SS])

$$
X=\left\{x \in R^{\nu N} \mid \sum m_{i} x_{i}=0\right\}
$$

with the inner product $\langle x, y\rangle=2 \sum m_{i} x_{i} \cdot y_{i}$. Here $m_{i}>0$ are masses of the particles in question. The Schrödinger operator of such a system is

$$
H=-\Delta+V(x) \text { on } L^{2}(X) \text {. }
$$

Here $\Delta$ is the Laplacian on $X$ and $V(x)=\sum V_{i j}\left(x_{i}-x_{j}\right)$, where $(i j)$ runs through all the pairs satisfying $i<j$. We assume that the potentials $V_{i j}$ are real and obey: $V_{i j}(y)$ are $\Delta_{y}$-compact. Under this condition, Kato's theorem applies and yields that $H$ is selfadjoint on $D(H)=D(\Delta)$ (see, e.g., [CFKS]). Moreover, if $V_{i j}$ are Kato potentials, i.e., $V_{i j} \in L^{r}\left(R^{\nu}\right)+\left(L^{\infty}\left(R^{\nu}\right)\right)_{\varepsilon}$, where $r>\frac{\nu}{2}$ if $\nu \geq 4$ and $r=2$ if $\nu \leq 3$, and the subscript $\varepsilon$ indicates that the $L^{\infty}$-component can be taken arbitrarily small, then $V_{i j}$ are Laplacian compact (see, e.g., [CFKS]).

We introduce the conditions on the potentials which are used in this paper.

(A) $|y|^{|\alpha|}\left|\partial^{\alpha} V_{i j}(y)\right|$ are $\Delta_{y}$-compact for $|\alpha|=0,1,2$.

(B) There is $R>0$ s.t. for $|y|>R, V_{i j} \in C^{2}$ and obey $\left|\partial^{\alpha} V(y)\right| \leq$ $C|y|^{-\mu-|\alpha|}$ with $|\alpha|=1,2$ and $\mu>0$.

In our analysis we will use an induction in number of particles. We will see later that already at the first inductive step one arrives at time-dependent Schrödinger operators of the form

$$
H(t)=H+W(t)
$$


where $H$ is a selfadjoint $N$-particle Schrödinger operator satisfying restrictions (A) and (B) and $W(t)$ is the multiplication operator by a real and smooth function $W(x, t)$ obeying

$$
\left|\partial_{(x, t)}^{\alpha} W(x, t)\right| \leq C_{\alpha}(1+|t|)^{-\mu-|\alpha|}
$$

with $\mu>0$ the same, though it is not necessary for our analysis, as in condition (B). Thus, guided by the consideration of induction convenience, we consider in this paper time-dependent Schrödinger operators described above. By $U(t)$ we denote the evolution operator generated by $H(t)$, i.e., the solution to the Cauchy problem

$$
i \frac{\partial}{\partial t} U(t)=H(t) U(t) \text { and } \quad U(0)=\mathrm{id} .
$$

We denote $\psi_{t}=U(t) \psi$.

The momentum space $X^{\prime}$, which is dual to $X$, is identified with (2.5)

$$
X^{\prime}=\left\{k \in R^{\nu N} \mid \sum k_{i}=0\right\} \quad \text { with the inner product }\langle k, u\rangle=\sum \frac{1}{2 m_{i}} k_{i} \cdot u_{i} \text {. }
$$

Thus $|k|^{2}$ is the symbol of $-\Delta$ and $-\Delta=|p|^{2}$ where $p=-\operatorname{grad}_{x}$. Using the natural bilinear form on $X \times X^{\prime},\langle x, k\rangle=\sum x_{i} \cdot k_{i}$, we introduce selfadjoint operators playing a prominent role in our analysis:

$$
A=\frac{1}{2}(\langle p, x\rangle+\langle x, p\rangle),
$$

the generator of dilations, and

$$
\gamma=\frac{1}{2}(\langle p, \hat{x}\rangle+\langle\hat{x}, p\rangle),
$$

the operator associated with the angle between the velocity and coordinate. Here $\hat{x}=\nabla(\langle x\rangle)$. Note that

$$
\gamma=\frac{1}{2} i[H,\langle x\rangle]
$$

Finally, we denote $E_{\Delta}=F(H \in \Delta)$ for a Borel set $\Delta \subset \mathbb{R}$.

\section{TIME-DEPENDENT OBSERVABLES}

In this section we outline some technical ideas used in this paper. By the time-dependent observable we usually mean a norm-differentiable family, $\phi(t)$, of selfadjoint bounded operators, which map $D(H)$ into itself. In those few cases when we use unbounded operators it is clear from the construction how to manipulate them. Define

$$
D \phi(t)=\frac{\partial \phi(t)}{\partial t}+i[H(t), \phi(t)] .
$$

We call this operation the Heisenberg derivative. (It is similar to the Lagrange derivative in Classical Mechanics.) Its property which warranted its introduction is $\frac{d}{d t}\langle\phi(t)\rangle_{t}=\langle D \phi(t)\rangle_{t}$, where $\langle\phi(t)\rangle_{t}=\left\langle\phi(t) \psi_{t}, \psi_{t}\right\rangle$. We will use the following refinement of this relation:

$$
\frac{d}{d t}\left\langle E_{\Delta} \phi(t) E_{\Delta}\right\rangle_{t}=\left\langle E_{\Delta} D \phi(t) E_{\Delta}\right\rangle_{t}+O\left(t^{-1-\mu}\right) .
$$


It follows if we observe that estimate (2.3) on $W(x, t)$ and an elementary commutator estimate of [SigSof4, Lemma A.1(i)] imply that

$$
\left[E_{\Delta}, W(x, t)\right]=O\left(\langle t\rangle^{-1-\mu}\right) .
$$

We will seek estimates of the form

$$
E_{\Delta} D \phi(t) E_{\Delta} \geq \theta E_{\Delta} F(t)^{2} E_{\Delta}-\sum F_{i}(t)^{2}
$$

where $\theta>0$ and $F$ and $F_{i}$ are time-dependent observables with $F_{i}$ satisfying

$$
\int_{0}^{\infty}\left\|F_{i}(t) \psi_{t}\right\|^{2} d t \leq C\|\psi\|^{2} \text { for all } i
$$

and for all $\psi \in \operatorname{Ran} E_{\Delta}$. Then the desired propagation estimate is given in the following:

Lemma 3.1. Let (3.3)-(3.4) hold. Let either (a) $E_{\Delta} \phi(t) E_{\Delta}$ be bounded uniformly in $t$ or (b) $E_{\Delta} \phi(t) E_{\Delta} \leq 0$. Then either

$$
\begin{aligned}
& \text { (a) } \int_{0}^{\infty}\left\|F(t) E_{\Delta} \psi_{t}\right\|^{2} d t \leq C\|\psi\|^{2} \quad \text { or } \\
& \text { (b) } \int_{0}^{\infty}\left\|F(t) E_{\Delta} \psi_{t}\right\|^{2} d t \leq-\left\langle\phi(0) E_{\Delta} \psi, E_{\Delta} \psi\right\rangle
\end{aligned}
$$

for all $\psi \in L^{2}$.

Proof. Integrating (3.1) in $t$ from 0 to $T$, we obtain

$$
\int_{0}^{T}\left\langle E_{\Delta} D \phi(t) E_{\Delta}\right\rangle_{t} d t=\left\langle E_{\Delta} \phi(T) E_{\Delta}\right\rangle_{T}-\left\langle E_{\Delta} \phi(0) E_{\Delta}\right\rangle_{0}
$$

Using inequalities (3.3) and (3.4) to estimate the 1.h.s. below and using the conditions of this lemma to estimate the r.h.s. above, we arrive at (3.5).

The most difficult task in this enterprise is to choose appropriate observables $\phi(t)$ and to prove for them estimates of the form (3.3)-(3.4). In this task we are aided by the following technical devices borrowed from [SigSof4] (see also [SigSof 3,6$]$ ). We consider smooth functions $f$ obeying

$$
\int_{-\infty}^{\infty}|s|^{n}|\hat{f}(s)| d s<\infty
$$

for some positive integer $n$. It is shown in [SigSof4, Lemma 1.1] (see also [SigSof 1, 3, 6]) that for $f$ obeying (3.7) with $n=2$ and a selfadjoint operator $B$ s.t. $B,[B, H]$, and $[B,[B, H]]$ are $H$-bounded and $[B,[B, H]]=O_{1}\left(R^{-2}\right)$, where $R$ is either $\langle x\rangle^{s}$ or $t^{s}$, the following relation holds:

$$
[H, f(B)]=[H, B] f^{\prime}(B)+O_{1}\left(R^{-2}\right) .
$$

Moreover, let $\phi(x)$ obey $\left|\partial^{\alpha} \phi(x)\right| \leq C_{\alpha} R^{-|\alpha|-\lambda}$ for $|\alpha| \leq 2$, where $\lambda \geq 0$. Then (see [SigSof1 (Lemmas A.4 and A.5), 3, 4])

$$
\begin{gathered}
{[f(\gamma), \phi(x)]=O\left(R^{-1-\lambda}\right),} \\
\sigma[f(\gamma), g(H / \sigma)]=O\left(\langle x\rangle^{-1}\right),
\end{gathered}
$$


and

$$
\sigma[\phi(x), g(H / \sigma)]=O\left(R^{-1-\lambda}\right),
$$

where the r.h.s.'s are uniform in $\sigma$.

Below we will use products of cut-off functions in $\gamma, \frac{x}{t}$, and $H$. Relations (3.9)-(3.11) render the order of the factors in such products immaterial for our purposes. Moreover, any such product differs by $O\left(t^{-s}\right)$ or by $O\left(\langle x\rangle^{-s}\right)$ with $s>0$ from its selfadjoint symmetric rearrangement, e.g., by placing square roots of cut-off functions symmetrically as in

$$
f(\gamma) g\left(\frac{\langle x\rangle}{t}\right)=f(\gamma)^{1 / 2} g\left(\frac{\langle x\rangle}{t}\right) f(\gamma)^{1 / 2}+O\left(t^{-1}\right),
$$

etc. Having this in mind we will manipulate with such products as if they are symmetrically rearranged. Thus, e.g., an inequality between two such products means, in fact, the corresponding inequality between their symmetrical rearrangements modulo $O\left(t^{-s}\right)$ or $O\left(\langle x\rangle^{-s}\right), s>0$.

For a linear subspace $R$ of $L^{2}(X)$ and a family $\phi(t)$ of bounded operators we define

$$
\phi(t) \doteq 0 \quad \text { on } R
$$

iff the inequality

$$
\int_{1}^{\infty}\left|\left\langle\phi(t) \psi_{t}, \psi_{t}\right\rangle\right| \frac{d t}{t} \leq C\|\psi\|^{2}
$$

holds for every $\psi \in R$. Here $C$ is independent of individual $\psi$ 's (but is dependent on $R$ ) and, recall, $\psi_{t}=U(t) \psi$.

\section{ASYMPTOTIC OBSERVABLES}

In this section we introduce asymptotic observables of interest and singular projections associated with them. We begin with some definitions. Let $\tau$ be the threshold set of $H$ (see $\S 8$ for the precise definition). An important fact about it is that it is a discrete and compact subset of $\mathbb{R}$. In fact, it has a finite number $(\leq N-1)$ of generations of accumulation points. For $\mathscr{A} \subset \mathbb{R}$ we set

$$
\Sigma_{\mathscr{A}}=\left\{(E-\lambda)^{1 / 2} \mid \lambda \in \tau, E \in \mathscr{A}, \lambda \leq E\right\} .
$$

For any $K \geq 0$, let $P^{K}$ be the orthogonal projection on the span of $K$ eigenfunctions of $H$ obeying

$$
P^{K} \rightarrow P \text { as } K \rightarrow \infty
$$

strongly. Here $P$ is the orthogonal projection on the pure point spectrum subspace of $H$. It is shown in [SigSof1, Proposition 7.2 and its proof] with extensions formulated and discussed in [SigSof6, §7] that for any discrete and compact set $\mathscr{A} \subset \mathbb{R}$ and any $\delta \notin \Sigma_{\mathscr{A}}$ there are $\delta_{0}>0, K>0$, and $\theta>0$ s.t.

$$
\begin{aligned}
E_{\Delta} i\left[H, F\left(\frac{\gamma}{\delta} \geq 1\right)\right] E_{\Delta} \leq & \frac{\theta}{\delta} E_{\Delta} \frac{1}{\sqrt{\langle x\rangle}} F^{\prime}\left(\frac{\gamma}{\delta} \geq 1\right) \frac{1}{\sqrt{\langle x\rangle}} E_{\Delta} \\
& -C \delta^{-1} E_{\Delta} \frac{1}{\sqrt{\langle x\rangle}} R \frac{1}{\sqrt{\langle x\rangle}} E_{\Delta}-C(\delta\langle x\rangle)^{-2}
\end{aligned}
$$


for any $\Delta \supset \mathscr{A}$ with $|\Delta| \leq \delta_{0}$ and any $F(\gamma / \delta \geq 1)$ satisfying

$$
\left|\operatorname{supp} F^{\prime}\left(\frac{s}{\delta} \geq 1\right)\right| \leq \delta_{0}|\delta|^{-1} \text {. }
$$

Here $C$ is independent of $\delta$ and $\langle x\rangle$ and

$$
R=F^{\prime}\left(\frac{\gamma}{\delta} \geq 1\right)^{1 / 2} P^{K} F^{\prime}\left(\frac{\gamma}{\delta} \geq 1\right)^{1 / 2} .
$$

In [SigSof1, Proposition 7.2] equation (4.3) is formulated for $\mathscr{A}=\{E\}$ away from the thresholds and eigenvalues of $H$, however the proof given there holds in the framework of (4.3). We sketch here its simplified version. [SigSof1, Theorem 6.1 (the channel expansion theorem) and Lemma 6.5] imply that for any $\varepsilon>0$ and any discrete and compact $\mathscr{A} \subset \mathbb{R}$ there are $\delta_{0}>0$ and $K \geq 0$ s.t. for any $\delta>0$

$$
\begin{aligned}
E_{\Delta} i[H, A] E_{\Delta} \geq & (\theta(\mathscr{A}, \delta)-\varepsilon)\left(E_{\Delta}\right)^{2} \\
& -C E_{\Delta} F\left(|\gamma| \leq \delta+\frac{2|\Delta|}{|\delta|}\right) E_{\Delta}-C E_{\Delta} P^{K} E_{\Delta}
\end{aligned}
$$

for any interval $\Delta \supset \mathscr{A}$ with $|\Delta| \leq \delta_{0}$ (see also [SigSof6, §7]). Here

$$
\theta(\mathscr{A}, \delta)=2 \min \left\{E-\lambda \mid \lambda \in \tau, E \in \mathscr{A}, E-\lambda \geq \delta^{2}\right\} .
$$

If $\delta \notin \Sigma_{\mathscr{A}}$, then, since $\Sigma_{\mathscr{A}}$ is a discrete set,

$$
\theta(\mathscr{A}, \delta)>2 \delta^{2} \text {. }
$$

Combining the last inequalities with (3.8) (with $B=\gamma$ and $R=\langle x\rangle$ ) and with

$$
i[H, \gamma]=\frac{1}{\sqrt{\langle x\rangle}}\left(i[H, A]-2 \gamma^{2}\right) \frac{1}{\sqrt{\langle x\rangle}}+O\left(\langle x\rangle^{-2}\right)
$$

and using commutation relations (3.10) and the localization property of $F^{\prime}(\gamma / \delta \geq 1)$, one arrives at (4.3).

We begin with a result parallel to that of [SigSof4, Theorem 4.1] on existence of asymptotic energy cut-offs:

Theorem 4.1. Let $\mathscr{A}$ be a discrete and compact subset of $\mathbb{R}, \frac{\varepsilon}{2} \notin \Sigma_{\mathscr{A}}$, and $\varepsilon>0$. Then there are $\delta_{0}, \delta_{1}>0$ s.t. the following strong limits exist

$$
F_{\varepsilon}^{ \pm}=\mathrm{s}-\lim _{t \rightarrow \pm \infty} U(t)^{*} E_{\Delta} F\left(\frac{\langle x\rangle}{\varepsilon|t|} \leq 1\right) E_{\Delta} U(t),
$$

provided $\Delta \supset \mathscr{A}$ and $|\Delta| \leq \delta_{0}$ and $\left|\operatorname{supp}\left(F^{\prime}\left(\frac{\lambda}{\varepsilon} \leq 1\right)\right)\right| \leq \delta_{1}$. Moreover, if $\varepsilon<\varepsilon^{\prime}$ and $\Delta \supset \Delta^{\prime}$ are s.t.

$$
F\left(\frac{\lambda}{\varepsilon^{\prime}} \leq 1\right) F\left(\frac{\lambda}{\varepsilon} \leq 1\right)=F\left(\frac{\lambda}{\varepsilon} \leq 1\right)
$$

and

$$
E_{\Delta^{\prime}}(\lambda) E_{\Delta}(\lambda)=E_{\Delta}(\lambda)
$$

then

$$
F_{\varepsilon^{\prime}}^{ \pm} F_{\varepsilon}^{ \pm}=F_{\varepsilon}^{ \pm}
$$

Proof. As usual we consider only the case $t \rightarrow+\infty$. We begin with 
Lemma 4.2. Let $\delta \notin \Sigma_{\mathscr{A}}$ and $\varepsilon>0$. Then there are $\delta_{0}, \delta_{1}>0$ s.t.

$$
F\left(\frac{\langle x\rangle}{\varepsilon t} \geq 1\right) F\left(\frac{\langle x\rangle}{\sigma t} \leq 1\right) F\left(\frac{\gamma}{\delta} \leq 1\right) \doteq 0,
$$

provided $2 \delta<\varepsilon$,

$$
F\left(\frac{\langle x\rangle}{\varepsilon t}=1\right) F\left(\frac{\langle x\rangle}{\sigma t} \leq 1\right) F\left(\frac{\gamma}{\delta} \geq 1\right) \doteq 0,
$$

provided $2 \delta>\varepsilon$, and

$$
F\left(\frac{\langle x\rangle}{\varepsilon t} \geq 1\right) F\left(\frac{\langle x\rangle}{\sigma t} \leq 1\right) F\left(\frac{\gamma}{\delta}=1\right) \doteq 0
$$

on $\operatorname{Ran} E_{\Delta}$, for any $\sigma \geq 1$ and for any $\Delta$ with $\Delta \supset \mathscr{A}$ and $|\Delta| \leq \delta_{0}$, provided $\left|\operatorname{supp} F\left(\frac{\lambda}{\delta}=1\right)\right|+\left|\operatorname{supp} F^{\prime}\left(\frac{\lambda}{\varepsilon} \geq 1\right)\right| \leq \delta_{1}$.

Proof. Since the proof of the first relation is a simplified version of the proof of the other two plus a covering argument (for the domain $\left\{\varepsilon \leq \frac{\langle x\rangle}{t} \leq \sigma\right\}$ by the sets $\left\{\left|\frac{\langle x\rangle}{t}-\nu\right| \leq \delta_{1}\right\}$ with $\left.\varepsilon \leq \nu \leq \sigma\right)$, we do here only the latter. Pick up $\sigma \geq 1$ so large that the maximal velocity bound

$$
F\left(\frac{\langle x\rangle}{\sigma t}=1\right) \doteq 0
$$

holds on $\operatorname{Ran} E_{\Omega}$, where $\Omega$ is a fixed bounded interval containing $\mathscr{A}$ (see [SigSof3, Theorem 4.3] and [SigSof4, Theorem 5.2]). Let first $2 \delta>\varepsilon$. We consider the propagation observable

$$
\phi=F_{1}\left(\frac{\langle x\rangle}{\varepsilon t} \geq 1\right) F_{2}\left(\frac{\langle x\rangle}{\sigma t} \leq 1\right) F_{3}\left(\frac{\gamma}{\delta} \geq 1\right) .
$$

We labeled the cut-off functions in order to simplify keeping track of them. Using (2.8) and (3.8), we compute its Heisenberg derivative

$$
\begin{aligned}
D \phi= & \frac{1}{\varepsilon t}\left(2 \gamma-\frac{\langle x\rangle}{t}\right) F_{1}^{\prime} F_{2} F_{3}+F_{1} \frac{1}{\sigma t}\left(2 \gamma-\frac{\langle x\rangle}{t}\right) F_{2}^{\prime} F_{3} \\
& +F_{1} F_{2} i\left[H, F_{3}\right]+O\left(t^{-2}\right) .
\end{aligned}
$$

Using that $2 \delta>\varepsilon$ and picking $\left|\operatorname{supp} F_{3}^{\prime}\left(\frac{s}{\delta} \geq 1\right)\right|+\left|\operatorname{supp} F_{1}^{\prime}\left(\frac{s}{\varepsilon} \geq 1\right)\right|<\delta-\frac{\varepsilon}{2}$, we conclude that the first term on the r.h.s. is

$$
\geq \theta_{1} t^{-1} F_{1}^{\prime} F_{2} F_{3}-C t^{-2} \text { with } \theta_{1}=\left(\delta-\frac{\varepsilon}{2}\right) \varepsilon^{-1}>0 \text {. }
$$

Next, equation (4.3) implies that there are $\delta_{0}, \delta_{1}$, and $K$ s.t. the third term on the r.h.s. sandwiched by $E_{\Delta}$ on each side is $\geq \theta_{2} t^{-1} E_{\Delta} F_{1} F_{2} F_{3}^{\prime} E_{\Delta}-C t^{-1} E_{\Delta} R_{1} E_{\Delta}$ for some $\theta_{2}>0$ and for any $\Delta$ with $\Delta \supset \mathscr{A}$ and $|\Delta| \leq \delta_{0}$, provided $\left|\operatorname{supp} F^{\prime}\left(\frac{\gamma}{\delta} \geq 1\right)\right| \leq \delta_{1}$. Here

$$
R_{1}=F_{1}^{1 / 2} F_{2}^{1 / 2}\left(F_{3}^{\prime}\right)^{1 / 2} P^{K}\left(F_{3}^{\prime}\right)^{1 / 2} F_{2}^{1 / 2} F_{1}^{1 / 2}
$$


These two conclusions together with the fact that $\gamma$ is $H$-bounded yield, under the above restrictions, that

$$
\begin{aligned}
E_{\Delta} D \phi E_{\Delta} \geq & \frac{\theta_{1}}{t} E_{\Delta} F_{1}^{\prime} F_{2} F_{3} E_{\Delta}+\frac{\theta_{2}}{t} E_{\Delta} F_{1} F_{2} F_{3}^{\prime} E_{\Delta} \\
& -\frac{C}{t} E_{\Delta} F_{1} F_{2}^{\prime} F_{3} E_{\Delta}-\frac{C}{t} E_{\Delta} R_{1} E_{\Delta}-\frac{C}{t^{2}} .
\end{aligned}
$$

In order to estimate the contribution by $R_{1}$ we use (3.9) to commute $F_{3}^{\prime 1 / 2}$ in the r.h.s. of (4.14) to the outside positions: $R_{1}=R_{2}+O\left(t^{-1}\right)$ with

$$
R_{2}=\left(F_{3}^{\prime}\right)^{1 / 2} F_{1}^{1 / 2} F_{2}^{1 / 2} P^{K} F_{2}^{1 / 2} F_{1}^{1 / 2}\left(F_{3}^{\prime}\right)^{1 / 2} \text {. }
$$

Now, denoting $\varphi(t)=\left(F_{3}^{\prime}\right)^{1 / 2} E_{\Delta} \psi_{t}$, we rewrite

$$
\int_{1}^{\infty}\left|\left\langle R_{2} \psi_{t}, \psi_{t}\right\rangle\right| \frac{d t}{t}=\int_{1}^{\infty}\left\|P^{K} F_{2}^{1 / 2} F_{1}^{1 / 2} \varphi(t)\right\|^{2} \frac{d t}{t}
$$

Write $P^{K} f=\sum_{\text {finite }} \psi^{\sigma}\left\langle\psi^{\sigma}, f\right\rangle$. Then

$$
\begin{aligned}
\int_{1}^{\infty}\left|\left\langle R_{2} \psi_{t}, \psi_{t}\right\rangle\right| \frac{d t}{t} & =\sum_{\text {finite }} \int_{1}^{\infty}\left|\left\langle\psi^{\sigma}, F_{2}^{1 / 2} F_{1}^{1 / 2} \varphi(t)\right\rangle\right|^{2} \frac{d t}{t} \\
& \leq C\|\psi\|^{2} \sum_{\text {finite }} \int_{1}^{\infty}\left\|F_{1}^{1 / 2} F_{2}^{1 / 2} \psi^{\sigma}\right\|^{2} \frac{d t}{t} .
\end{aligned}
$$

Since

$$
\begin{aligned}
\int_{1}^{\infty}\left\|F_{1}^{1 / 2} F_{2}^{1 / 2} \psi^{\sigma}\right\|^{2} \frac{d t}{t} & \leq \iint_{\frac{\varepsilon}{2} t \leq\langle x\rangle \leq 2 \sigma t, t \geq 1}\left|\psi^{\sigma}(x)\right|^{2} \frac{d x d t}{t} \\
& =\ln \left(\frac{4 \sigma}{\varepsilon}\right)\left\|\psi^{\sigma}\right\|^{2}
\end{aligned}
$$

we conclude that

$$
\int_{1}^{\infty}\left|\left\langle R_{1} \psi_{t}, \psi_{t}\right\rangle\right| \frac{d t}{t} \leq C\|\psi\|^{2} .
$$

Integrating differential inequality (4.15) and using (3.1), (4.11), and (4.18), we conclude that $(4.10 \mathrm{~b})$ holds on $\operatorname{Ran} E_{\Delta}$, provided $2 \delta>\varepsilon$. Since for any $\varepsilon_{1}>0$ there is $\varepsilon<\varepsilon_{1}$ satisfying $0<\varepsilon<2 \delta$ and

$$
F\left(\frac{\langle x\rangle}{\varepsilon_{1} t} \geq 1\right)=F\left(\frac{\langle x\rangle}{\varepsilon_{1} t} \geq 1\right) F\left(\frac{\langle x\rangle}{\varepsilon t} \geq 1\right),
$$

we conclude that (4.10b) holds for any $\varepsilon>0$ and any $\delta \notin \Sigma_{\mathscr{A}}$.

This lemma implies readily

Corollary 4.3. Let $\mathscr{A}$ be a discrete and compact subset of $\mathbb{R}$ and $\frac{\varepsilon}{2} \notin \Sigma_{\mathscr{A}}$. Then there are $\delta_{0}, \delta_{1}>0$ s.t.

$$
F\left(\frac{\langle x\rangle}{\varepsilon t}=1\right) \doteq 0
$$


on $\operatorname{Ran} E_{\Delta}$, for any $\Delta$ with $\Delta \supset \mathscr{A}$ and $|\Delta| \leq \delta_{0}$ and for any cut-off function obeying $\left|\operatorname{supp} F\left(\frac{\lambda}{\varepsilon}=1\right)\right| \leq \delta_{1}$.

Now we return to the proof of Theorem 4.1. Using (3.1), we obtain

$$
\begin{aligned}
& \frac{d}{d t} U(t)^{*} E_{\Delta} F\left(\frac{\langle x\rangle}{\varepsilon t} \leq 1\right) E_{\Delta} U(t) \\
& \quad=U(t) E_{\Delta} D F\left(\frac{\langle x\rangle}{\varepsilon t} \leq 1\right) E_{\Delta} U(t)+O\left(t^{-1-u}\right) .
\end{aligned}
$$

Moreover, using, as before, (2.8) and (3.8), we find

$$
D F\left(\frac{\langle x\rangle}{\varepsilon t} \leq 1\right)=-\frac{1}{\varepsilon t}\left(2 \gamma-\frac{\langle x\rangle}{\varepsilon t}\right) F^{\prime}\left(\frac{\langle x\rangle}{\varepsilon t} \leq 1\right)+O\left((\varepsilon t)^{-2}\right) .
$$

Using that $F^{\prime}\left(\frac{\lambda}{\varepsilon} \leq 1\right)=c F\left(\frac{\lambda}{\varepsilon}=1\right)$, where $c>0$, using that $\gamma$ is $H$-bounded, and using (4.19), we arrive at

$$
\int_{1}^{T}\left|\frac{d}{d t}\left\langle v, U(t)^{*} E_{\Delta}\left(D F\left(\frac{\langle x\rangle}{\varepsilon t} \leq 1\right)\right) E_{\Delta} U(t) u\right\rangle\right| d t \leq C\|u\|\|v\|
$$

for any $u, v \in L^{2}$ and with $C$ independent of the $u$ 's and $v$ 's. The latter inequality implies that $U(t)^{*} E_{\Delta} F\left(\frac{\langle x\rangle}{\varepsilon t} \leq 1\right) E_{\Delta} U(t)$ converges strongly as $t \rightarrow$ $+\infty$. This proves (4.5).

Now we show (4.8). By (4.5) and by (4.7)

$$
F_{\varepsilon^{\prime}}^{ \pm} F_{\varepsilon}^{ \pm}=\mathrm{s}-\lim _{t \rightarrow \pm \infty} U(t)^{*} E_{\Delta^{\prime}} F_{\varepsilon^{\prime}} E_{\Delta} F_{\varepsilon} E_{\Delta} U(t),
$$

where $F_{\varepsilon}=F\left(\frac{\langle x\rangle}{t} \leq \varepsilon\right)$. Let $\bar{F}_{\varepsilon^{\prime}}=1-F_{\varepsilon^{\prime}}$. By (4.6) and by (3.11)

$$
\bar{F}_{\varepsilon^{\prime}} E_{\Delta} F_{\varepsilon}=O\left(t^{-1}\right) \text {. }
$$

This together with (4.7) and (4.21) yields (4.8).

Below by $\varepsilon \rightarrow 0$ we mean that, given a discrete and compact set $\mathscr{A}, \varepsilon$ approaches 0 along the set $\mathbb{R} \backslash \Sigma_{\mathscr{A}}$ (which is possible since $\Sigma_{\mathscr{A}}$ is discrete). We emphasize that in the definition of $F_{\varepsilon}^{ \pm}$, equation (4.4), the energy interval $\Delta$ depends on $\varepsilon$, while $\varepsilon \notin \Sigma_{\mathscr{A}}$. The next theorem introduces singular projections associated with $\langle x\rangle / t$. It is similar to [SigSof6, Theorem 4.1] (cf. also [SigSof4, Theorem 4.1(ii)]).

Theorem 4.4. The bounded operators

$$
F^{ \pm}=\mathrm{s}-\lim _{\varepsilon \downarrow 0} F_{\varepsilon}^{ \pm}
$$

exist and obey

$$
\begin{gathered}
\left(F^{ \pm}\right)^{2}=F^{ \pm}, \\
\left(F^{ \pm}\right)^{*}=F^{ \pm} \geq 0,
\end{gathered}
$$

and for any $\varepsilon>0$

$$
F_{\varepsilon}^{ \pm} F^{ \pm}=F^{ \pm} .
$$


Proof. Note that $F_{\varepsilon}^{ \pm} \geq 0$ and are monotonically nonincreasing as $\varepsilon \downarrow 0$. Hence there are $F^{ \pm} \geq 0$ s.t. $F_{\varepsilon}^{ \pm} \downarrow F^{ \pm}$and $\varepsilon \downarrow 0$, weakly. Picking $\varepsilon^{\prime}, \Delta^{\prime}, \varepsilon$, and $\Delta$ so that (4.6) and (4.7) hold and using (4.8), we find

$$
\left\|\left(F_{\varepsilon^{\prime}}^{ \pm}-F_{\varepsilon}^{ \pm}\right) u\right\|^{2}=\left\|F_{\varepsilon^{\prime}}^{ \pm} u\right\|^{2}+\left\|F_{\varepsilon}^{ \pm} u\right\|^{2}-2\left\langle F_{\varepsilon}^{ \pm} u, u\right\rangle \text {. }
$$

Since $0 \leq F_{\varepsilon}^{ \pm} \leq 1$, we find furthermore

$$
\begin{aligned}
\left\|\left(F_{\varepsilon^{\prime}}^{ \pm}-F_{\varepsilon}^{ \pm}\right) u\right\|^{2} & \leq\left\|\left(F_{\varepsilon^{\prime}}^{ \pm}\right)^{1 / 2} u\right\|^{2}+\left\|\left(F_{\varepsilon}^{ \pm}\right)^{1 / 2} u\right\|-2\left\langle F_{\varepsilon}^{ \pm} u, u\right\rangle \\
& =\left\langle F_{\varepsilon^{\prime}}^{ \pm} u, u\right\rangle-\left\langle F_{\varepsilon}^{ \pm} u, u\right\rangle \rightarrow 0
\end{aligned}
$$

as $\varepsilon^{\prime}, \varepsilon \rightarrow 0$. Hence $F_{\varepsilon}^{ \pm} \rightarrow F^{ \pm}$strongly as $\varepsilon \rightarrow 0$. (4.25) is obvious and (4.24) and (4.26) follow from (4.23) and (4.8).

Next, we need the asymptotic energy cut-offs of [SigSof6]. That paper states that the limits

$$
E_{\Delta}^{ \pm}=\mathrm{s}-\lim _{t \rightarrow \pm \infty} U(t)^{*} E_{\Delta} U(t)
$$

exist for any open Borel set $\Delta$. Moreover, if $\mathscr{A}$ is a Borel set of measure 0 , then

$$
Q_{\mathscr{A}}^{ \pm}=\mathrm{s}-\lim _{\Delta \rightarrow \mathscr{A}} E_{\Delta}^{ \pm}
$$

where the limit is taken over a sequence of open sets containing $\mathscr{A}$ and shrinking to it, exist and are independent of the sequence of $\Delta$ 's taken. It is shown in [SigSof6] that $Q_{\mathscr{A}}^{ \pm}$are selfadjoint projections and that $Q_{\mathscr{A}}^{ \pm} Q_{\mathscr{B}}^{ \pm}=Q_{\mathscr{A} \cap \mathscr{B}}^{ \pm}$(this is not used in this paper) and

$$
\left\|\psi_{t}-E_{\Delta} \psi_{t}\right\| \leq C|\Delta|^{-1} t^{-\mu}
$$

for any $\Delta$ containing $\mathscr{A}$, provided $\psi \in \operatorname{Ran} Q_{\mathscr{A}}^{ \pm}$. Here $C$ is independent of $t$ and of $\Delta$ (the independence of $\Delta$ is not used in this paper). Property (4.28) and Theorem 4.4 yield

Theorem 4.5. Let $\mathscr{A}$ be a discrete and compact subset of $\mathbb{R}$. Then the limits

$$
F_{\varepsilon}^{ \pm}=\mathrm{s}-\lim _{t \rightarrow \pm \infty} U(t)^{*} F\left(\frac{\langle x\rangle}{\varepsilon|t|} \leq 1\right) U(t)
$$

with $\varepsilon>0$ and $\varepsilon \notin \Sigma_{\mathscr{A}}$, and

$$
F^{ \pm}=\mathrm{s}-\lim _{\varepsilon \downarrow 0} F_{\varepsilon}^{ \pm}
$$

exist on $\operatorname{Ran} Q_{\mathscr{A}}^{ \pm}$. Moreover, these limits coincide (on $\operatorname{Ran} Q_{\mathscr{A}}^{ \pm}$, of course) with (4.5) and (4.23), respectively.

We will also need the following

Theorem 4.6. Operators $Q_{\mathscr{A}}^{+}$and $F^{+}$(resp. $Q_{\mathscr{A}}^{-}$and $F^{-}$) commute.

Proof. By the definition, by a property of strong convergence and by uniform boundedness of $F_{\varepsilon}^{+}$and $E_{\Delta}^{+}$,

$$
\left[F^{+}, Q_{\mathscr{A}}^{+}\right]=\mathrm{s}-\lim _{\substack{\varepsilon \rightarrow 0 \\ \Delta \rightarrow \mathscr{A}}}\left[F_{\varepsilon}^{+}, E_{\Delta}^{+}\right],
$$


where the limits can be taken in any order. Again, by a property of strong convergence and uniform boundedness of $U(t)^{*} E_{\Delta_{1}} F\left(\frac{\langle x\rangle}{\varepsilon t} \leq 1\right) E_{\Delta_{1}} U(t)$ and of $U(t)^{*} E_{\Delta} U(t)$

$$
\left[F_{\varepsilon}^{+}, E_{\Delta}^{+}\right]=\lim _{t \rightarrow+\infty} U(t)^{*} E_{\Delta_{1}}\left[F\left(\frac{\langle x\rangle}{\varepsilon t} \leq 1\right), E_{\Delta}\right] E_{\Delta_{1}} U(t) .
$$

By (3.11) with $R=t$, the commutator on the r.h.s. is $O\left(t^{-1}\right)$. Hence $\left[F_{\varepsilon}^{+}, E_{\Delta}^{+}\right]$ $=0$, which yields $\left[F^{+}, Q_{\mathscr{A}}^{+}\right]=0$.

We denote $Q^{ \pm}=Q_{\mathscr{A}}^{ \pm}$whenever $\mathscr{A}$ is the threshold set, $\tau$, of $H$.

Theorem 4.7. $\operatorname{Ran} F^{ \pm} \subseteq \operatorname{Ran} Q^{ \pm}$.

Proof. Let $\psi \in\left(\operatorname{Ran} Q^{+}\right)^{\perp}$. Then, by the definition of $Q^{+}$, for any $\varepsilon>0$ there is a closed set $\Omega$ disjoint from $\tau$ and s.t.

$$
\left\|\psi-E_{\Omega}^{+} \psi\right\| \leq \varepsilon .
$$

By the minimal velocity estimate ([SigSof4, Theorem 5.1], see also [SigSof3, Theorem 4.2]) there is $\delta>0$ s.t.

$$
\left\|F\left(\frac{\langle x\rangle}{t} \leq \delta\right) E_{\Omega} \psi_{t}\right\| \rightarrow 0
$$

as $t \rightarrow+\infty$. In terms of asymptotic cut-offs this can be rewritten as $F_{\delta}^{+} E_{\Omega}^{+} \psi=$ 0 , i.e., $E_{\Omega}^{+} \psi \in\left(\operatorname{Ran} F_{\delta}^{+}\right)^{\perp} \subset\left(\operatorname{Ran} F^{+}\right)^{\perp}$. Taking this into account and remembering that $\varepsilon>0$ in (4.29) is arbitrary we obtain that $\psi \in\left(\operatorname{Ran} F^{+}\right)^{\perp}$.

\section{Microlocal ANALYSis ON $\operatorname{Ran} F^{ \pm}$}

In this section we show that $\langle x\rangle$ is sharply localized on $\operatorname{Ran} F^{ \pm}$. We begin with

Theorem 5.1. Let $\delta>0$ and $\varepsilon>0$. Then there is $\Delta \supset \tau$ s.t. for any $\alpha \in(0,1]$ and any $\psi \in \operatorname{Ran} F^{+}$we have

$$
\left\|F\left(t^{1-\alpha} \frac{\gamma}{\delta} \geq 1\right) F\left(\frac{\langle x\rangle}{\varepsilon t^{\alpha}} \geq 1\right) E_{\Delta} \psi_{t}\right\|^{2} \leq C t^{3-2 \alpha-\eta},
$$

where $\eta=\left(1-2^{-N-1}\right)^{-1}$.

Proof. Let $\gamma_{t}$ be the selfadjoint operator related to $\gamma$ and defined in Theorem A.1. Let first $\delta \notin \Sigma_{\mathscr{A}}$ and $\varepsilon<2 \delta$. Consider the propagation observable

$$
\phi=F\left(\frac{\langle x\rangle}{\varepsilon t} \geq 1\right) F\left(\frac{\gamma t}{\delta} \geq 1\right) F\left(\frac{\langle x\rangle}{\varepsilon t} \geq 1\right) .
$$

In the proof below $O(\cdots)$ and $O_{1}(\cdots)$ stand for an estimate uniform in $\varepsilon$ and $\delta$. Using equation (3.8), we compute its Heisenberg derivative

$$
\begin{aligned}
D \phi= & (\varepsilon t)^{-1}\left(2 \gamma-\frac{\langle x\rangle}{t}\right) F_{1}^{\prime} F_{2} F_{1}+\text { adjoint } \\
& +F_{1} D F_{2} F_{1}+O\left((\varepsilon t)^{-2}\right)
\end{aligned}
$$


where we have used our standard abbreviations for cut-off functions and where we have used that

$$
\langle x\rangle^{-s} F\left(\frac{\langle x\rangle}{\varepsilon t} \geq 1\right)=O\left((\varepsilon t)^{-s}\right) .
$$

Since $2 \delta>\varepsilon$, we can choose the cut-off functions so that

$$
2 \inf \operatorname{supp} F_{2}\left(\frac{\lambda}{\delta} \geq 1\right)-\sup \operatorname{supp} F_{1}^{\prime}\left(\frac{\lambda}{\varepsilon} \geq 1\right)>0 \text {. }
$$

Thus, due to (A.1), the first two terms on the r.h.s. are

$$
\geq \frac{\theta_{1}}{\varepsilon t} \operatorname{Re}\left(F_{1}^{\prime} F_{2} F_{1}\right)-C(\varepsilon t)^{-2}
$$

for some $\theta_{1}>0$. Next, using first (3.8), with $B=\gamma_{t}$ and $R=t^{-\eta / 2}$, then symmetrizing with the help of estimates of the type of (3.10) and (A.2), we obtain

$$
D F_{2}=\frac{1}{\delta}\left(F_{2}^{\prime}\right)^{1 / 2} D \gamma_{t}\left(F_{2}^{\prime}\right)^{1 / 2}+O_{1}\left(\delta^{-2} t^{-\eta}\right)
$$

Now using (A.3), we derive

$$
D F_{2} \geq O_{1}\left(\delta^{-2} t^{-\eta}\right)
$$

This together with (5.3) and (5.6) yields

$$
D \phi \geq O_{1}\left(\varepsilon^{-2} t^{-\eta}\right)
$$

Now we introduce, for $\Delta$ the same as in Lemma 4.2,

$$
\phi_{\Delta}^{t}=U(t)^{*} E_{\Delta} \phi E_{\Delta} U(t) .
$$

Combining this with a more precise version, due to (3.11), of (3.1), we obtain

$$
\frac{d}{d t} \phi_{\Delta}^{t}=U(t)^{*} E_{\Delta} D \phi E_{\Delta} U(t)+O\left(|\Delta|^{-1} t^{-1-\mu}\right) .
$$

Due to equation (A.1) we can replace $\gamma$ by $\gamma_{t}$ in the statement of Lemma 4.2. This and equation (5.3) yield that for any $\sigma>0$

$$
\int_{1}^{\infty}\left|\left\langle E_{\Delta} u_{t},(D \phi) F\left(\frac{\langle x\rangle}{\sigma t} \leq 1\right) E_{\Delta} v_{t}\right\rangle\right| d t \leq C\|u\|\|v\| .
$$

Next we need the maximal velocity estimate of [SigSof3, Theorem 4.3] (or [SigSof4, Theorem 3.3]): for any bounded $\Omega$, for $\sigma>0$ sufficiently large (depending on $\Omega$ ), and for any $\psi \in L^{2}$

$$
\int_{1}^{\infty}\left\|F\left(\frac{\langle x\rangle}{\sigma t} \geq 1\right) E_{\Omega} \psi_{t}\right\|^{2} t^{-\rho} d t \leq C\left\|\langle x\rangle^{1 / 2} \psi\right\|^{2},
$$

where $\rho>\frac{\text { const }}{\sigma}$ with the constant positive and depending only on $\Omega$. The last three estimates and the fact that $D \phi=O_{1 / 2}\left(t^{-1}\right)$, yield

$$
\int_{1}^{\infty}\left|\frac{d}{d t}\left\langle\phi_{\Delta}^{t} \psi, u\right\rangle\right| d t \leq C\left\|\langle x\rangle^{1 / 2} \psi\right\|\|u\|,
$$


which implies that

$$
\phi^{\infty}=\mathrm{s}-\lim _{t \rightarrow+\infty} \phi_{\Delta}^{t}
$$

exist on $\langle x\rangle^{-1 / 2} L^{2}$ and therefore on $L^{2}$. Now let $\phi^{t}=U(t)^{*} \phi U(t)$. By (4.28) and (3.10)-(3.11) (with $R=\varepsilon t$ )

$$
\phi^{t}=\phi_{\Delta}^{t}+O\left((|\Delta| \delta \varepsilon t)^{-1}\right)+O\left(|\Delta|^{-1} t^{-\mu}\right)
$$

on $\operatorname{Ran} Q^{+}$. Hence

$$
\phi^{\infty}=\mathrm{s}-\lim _{t \rightarrow+\infty} \phi^{t}
$$

exists on $\operatorname{Ran} Q^{+}$. Now combining

$$
\phi^{t}-\phi^{\infty}=-\int_{t}^{\infty} \frac{d}{d s} \phi^{s} d s
$$

with (5.8) and with the relation $\frac{d}{d s} \phi^{s}=U(s)^{*} D \phi U(s)$, we obtain that on $\operatorname{Ran} Q^{+}$

$$
\phi^{t} \leq \phi^{\infty}+C \varepsilon^{-2} t^{1-\eta} .
$$

Note now that by the definition of $F^{+}$and a property of strong convergence

$$
\phi^{\infty} \psi=0 \text { for any } \psi \in \operatorname{Ran} F^{+} \text {. }
$$

The last two relations and the definition of $\phi^{t}$ yield

$$
\left\|F\left(\frac{\gamma}{\delta} \geq 1\right) F\left(\frac{\langle x\rangle}{\varepsilon t} \geq 1\right) \psi_{1}\right\|^{2} \leq C \varepsilon^{-2} t^{1-\eta},
$$

provided $\psi \in \operatorname{Ran} F^{+}\left(\subset \operatorname{Ran} Q^{+}\right), \delta \notin \Sigma_{\tau}$, and $\varepsilon<2 \delta$. Clearly, the last two restrictions on $\delta$ and $\varepsilon$ can be dropped. Replacing $\varepsilon$ by $\varepsilon t^{\alpha-1}$ and $\delta$ by $\delta t^{\alpha-1}$ in (5.15), we arrive at (5.1).

Now we consider the remaining part of phase-space. The results which follow are closely related to results of [SigSof6, §12].

Theorem 5.2. Let $\alpha>\frac{3-\eta}{2}, \varepsilon>0$, and $\delta<\frac{\alpha \varepsilon}{2}$. Then

$$
F\left(\frac{\langle x\rangle}{t^{\alpha}}=\varepsilon\right) F\left(t^{1-\alpha} \gamma \leq \delta\right) \doteq 0
$$

on $\operatorname{Ran} E_{\Omega}$ for any bounded $\Omega$.

Proof. Consider the propagation observable

$$
\phi=F\left(\frac{\langle x\rangle}{t^{\alpha}} \geq \varepsilon\right) F\left(t^{1-\alpha} \gamma_{t} \leq \delta\right)
$$

with the parameter specified in the theorem. Using (3.8) and (A.2), we compute its Heisenberg derivative

$$
\begin{aligned}
D \phi= & \frac{1}{t}\left(t^{1-\alpha} 2 \gamma-\alpha \frac{\langle x\rangle}{t^{\alpha}}\right) F^{\prime} F \\
& +F t^{1-\alpha} D \gamma_{t} F^{\prime}+O\left(t^{-2 \alpha}\right)+O_{1}\left(t^{1-\alpha-\eta}\right) .
\end{aligned}
$$


Using (A.1) (and the fact that $\eta_{1}>1-\alpha$ ) and the support properties of the cut-off functions, and choosing these functions sufficiently sharp, we conclude that the first term on the r.h.s is $\leq-\theta t^{-1} F^{\prime} F$ for some $\theta>0$. Symmetrizing the second term on the r.h.s. and using (A.2) and (A.3); we find that it is $\leq$ $O_{1}\left(t^{2-2 \alpha-\eta}\right)$. Thus

$$
D \phi \leq-\theta t^{-1} F^{\prime} F+C t^{-2 \alpha}+O_{1}\left(t^{2-2 \alpha-\eta}\right) .
$$

By the condition on $\alpha$ the second and third terms on the r.h.s. are integrable. Applying the first part of Lemma 3.1 to this differential inequality and using that $F^{\prime}\left(\frac{\langle x\rangle}{t^{\alpha}} \geq 1\right)=$ const $\cdot F\left(\frac{\langle x\rangle}{t^{\alpha}}=1\right)$ with a positive constant, we arrive at $F\left(\frac{\langle x\rangle}{t^{\alpha}}=\varepsilon\right) F\left(t^{1-\alpha} \gamma_{t} \leq \delta\right) \doteq 0$ on $\operatorname{Ran} E_{\Omega}$ for any bounded $\Omega$. Finally, using (A.1) we derive readily that

$$
F\left(t^{1-\alpha} \gamma_{t} \leq \delta\right)=F\left(t^{1-\alpha} \gamma \leq \delta\right)+O\left(t^{1-\alpha-\eta_{1}}\right),
$$

where $\eta_{1}=2^{-N-1}$. Since $\eta_{1}>1-\alpha$, this allows us to pass from the last statement to $(5.16)$.

Theorem 5.3. Let $\frac{3 \eta}{2}<\alpha \leq 1, \varepsilon>0, \delta<\frac{\alpha \varepsilon}{2}$, and

$$
0 \leq \rho<\min \left(2 \alpha+\eta-3, \alpha+\eta_{1}-1\right) \text {. }
$$

Let $\Omega$ be bounded. Then

$$
\int_{1}^{\infty}\left\|F\left(\frac{\langle x\rangle}{t^{\alpha}} \geq \varepsilon\right) F\left(t^{1-\alpha} \gamma \leq \delta\right) E_{\Omega} \psi_{t}\right\|^{2} \frac{d t}{t^{1-\rho}} \leq C\left\|\langle x\rangle^{1 / 2} \psi\right\|^{2}
$$

for any $\psi \in L^{2}$ and with $C$ independent of the $\psi$ 's.

Proof. For given $\Omega$ we pick up $\sigma>0$ so large that (5.11) holds. Let $f(\lambda)=$ $\lambda F(\lambda \geq \varepsilon)$. With the parameters defined in the lemma we introduce the observable

$$
\phi=-t^{\rho} f\left(\frac{\langle x\rangle}{t^{\alpha}}\right) F\left(\frac{\langle x\rangle}{t} \leq \sigma\right) F\left(t^{1-\alpha} \gamma_{t} \leq \delta\right),
$$

where, recall, $\gamma_{t}$ is given in Theorem A.1. Compute the Heisenberg derivative

$$
\begin{aligned}
D \phi= & t^{\rho-1}\left(\alpha \frac{\langle x\rangle}{t^{\alpha}}-\frac{2 \gamma}{t^{\alpha-1}}\right) F^{\prime} F F-\rho t^{\rho-1} f F F \\
& -t^{\rho} f D F F-t^{\rho} f F t^{1-\alpha}\left(D \gamma_{t}\right) F^{\prime}+O_{1}\left(t^{\rho+1-\alpha-\eta}\right)+O\left(t^{\rho-2 \alpha}\right) .
\end{aligned}
$$

Symmetrizing and using (A.2) and (A.3), we estimate the fourth term on the r.h.s. from below by $O_{1}\left(t^{\rho+2-2 \alpha-\eta}\right)$. Next, using support properties of the first and third cut-off functions and using that $f(\lambda) \leq \lambda f^{\prime}(\lambda)$, we estimate the first two terms on the r.h.s. from below by $t^{-1}\left((\alpha-\rho) \varepsilon-2 \delta-\delta_{1}\right) f^{\prime} F F$, where $\delta_{1}=\left|\operatorname{supp} F^{\prime}(\lambda \geq \varepsilon)\right|+\left|\operatorname{supp} F^{\prime}(\lambda \leq \delta)\right|$. Picking up $\delta_{1} \leq \frac{1}{2}((\alpha-\rho) \varepsilon-2 \delta) \equiv \eta$ and using that $f(\lambda) \geq \varepsilon F(\lambda \geq \varepsilon)$, we conclude that

$$
D \phi \geq \eta t^{\rho-1} F F F+f D F F+O_{1}\left(t^{\rho+2-2 \alpha-\eta}\right) .
$$


Next we have

$$
D F\left(\frac{\langle x\rangle}{t} \leq \sigma\right)=t^{-1}\left(2 \gamma-\frac{\langle x\rangle}{t}\right) F^{\prime}\left(\frac{\langle x\rangle}{t} \leq \sigma\right)+O\left(t^{-2}\right) .
$$

Using now that $F^{\prime}\left(\frac{\langle x\rangle}{t} \leq \sigma\right)=F\left(\frac{\langle x\rangle}{t}=\sigma\right)$, times a positive constant, that $\gamma$ is $\sqrt{-\Delta}$-bounded, and that $\phi \leq 0$, and following the second option in Lemma 3.1, we derive from (5.23), (5.24), and (5.11) that

$$
\begin{aligned}
& \int_{1}^{\infty}\left\|F\left(\frac{\langle x\rangle}{t^{\alpha}} \geq \varepsilon\right) F\left(\frac{\langle x\rangle}{t} \leq \sigma\right) F\left(t^{1-\alpha} \gamma_{t} \leq \rho\right) E_{\Omega} \psi_{t}\right\|^{2} \frac{d t}{t^{1-\rho}} \\
& \quad \leq C\left\|\langle x\rangle^{1 / 2} \psi\right\|^{2} .
\end{aligned}
$$

Combining with this (5.11) and using (5.19) in order to pass from $\gamma_{t}$ to $\gamma$, we obtain (5.20).

Theorem 5.4. Let $\alpha>\frac{3-\eta}{2}, \varepsilon>0$, and $\delta<\frac{\alpha \varepsilon}{2}$. Let $\Omega$ be bounded. Then for any $\psi \in L^{2}$ and as $t \rightarrow+\infty$

$$
F\left(\frac{\langle x\rangle}{t^{\alpha}} \geq \varepsilon\right) F\left(t^{1-\alpha} \gamma \leq \delta\right) E_{\Omega} \psi_{t} \rightarrow 0 \text {. }
$$

Proof. Let $\phi_{t}$ be given by (5.17). Equations (5.20) and (5.19) imply that there is a sequence $t_{n}$ s.t. $t_{n} \rightarrow \infty$ and

$$
\phi_{t_{n}} \psi_{t_{n}} \rightarrow 0
$$

as $n \rightarrow \infty$ for any $\psi \in L^{2}$. Consider now the Heisenberg family

$$
\phi^{t}=U(t)^{*} E_{\Omega} \phi_{t}^{*} \phi_{t} E_{\Omega} U(t) .
$$

Observe that, due to (3.9) with $R=t^{\alpha}$ and $\lambda=0, \phi_{t}^{*} \phi_{t}$ is, modulo $O\left(t^{1-2 \alpha}\right)$, of the form (5.17). In fact, an estimate of the type of (5.18) can be shown directly for $\phi_{t}^{*} \phi_{t}$. Hence, by (3.1) and (5.18)

$$
\frac{d}{d t} \phi^{t} \leq C t^{-1-\mu}+O_{1}\left(t^{2-2 \alpha-\eta}\right) \text {. }
$$

Since, by our assumptions on $\alpha$, the r.h.s. is integrable and since $\phi^{t} \geq 0$, we obtain that $\left\langle\phi^{t} \psi, \psi\right\rangle=\left\|\phi_{t} E_{\Omega} \psi_{t}\right\|^{2}$ converges as $t \rightarrow+\infty$. This together with (5.27) yields that $\phi_{t} E_{\Omega} \psi_{t} \rightarrow 0$ as $t \rightarrow \infty$. Finally, using (5.19), we can pass in this relation from $\gamma_{t}$ to $\gamma$ to arrive at (5.26).

Corollary 5.5. Let $\alpha>\frac{3-\eta}{2}$. Then for any $\psi \in \operatorname{Ran} F^{+}$

$$
F\left(\frac{\langle x\rangle}{t^{\alpha}} \geq 1\right) \psi_{t} \rightarrow 0 \text { as } t \rightarrow+\infty \text {. }
$$

Proof. Combining Theorems 5.1 and 5.5, we obtain

$$
F\left(\frac{\langle x\rangle}{t^{\alpha}} \geq 1\right) E_{\Omega} \psi_{t} \rightarrow 0
$$


as $t \rightarrow+\infty$, for any bounded $\Omega$. On the other hand by [SigSof4, Theorem 4.1(ii)] for any $\delta>0$ there is a bounded interval $\Omega$ s.t.

$$
\sup \left\|\left(1-E_{\Omega}\right) \psi_{t}\right\| \leq \delta .
$$

The last two relations yield (5.30).

\section{REDUCTION}

In this section we show that $\psi_{t}$, for any $\psi \in \operatorname{Ran} F^{+}$, approaches as $t \rightarrow+\infty$ an orbit generated, modulo a time-dependent factor, by the time-independent Hamiltonian $H$. Indeed, if

$$
U_{0}(t)=e^{-i H t} e^{-i \int_{0}^{t} W(0, s) d s},
$$

then the difference between the generators of $U(t)$ and $U_{0}(t)$, i.e., $W(x, t)-$ $W(0, t)$, is $O\left(t^{-1-\mu+\alpha}\right)$ in the region $|x| \leq C t^{\alpha}$ in which $\psi_{t}$ is localized for large $t$. Therefore it is integrable for $\alpha<\mu$. In this section we follow closely the arguments of [SigSof6, §5].

To cast the discussion above into rigorous terms we introduce the wave operators

$$
W_{0}^{ \pm}=\mathrm{s}-\lim _{t \rightarrow \pm \infty} U_{0}(t)^{*} F\left(\frac{\langle x\rangle}{|t|^{\alpha}} \leq 1\right) U(t)
$$

whenever they exist.

Theorem 6.1. Let $\mu>\alpha>\frac{3-\eta}{2}$. Then $W_{0}^{ \pm}$exist on $\operatorname{Ran} F^{ \pm}$.

Proof. Differentiating the family $W_{0}(s) \equiv U_{0}(s)^{*} F\left(\frac{\langle x\rangle}{s^{\alpha}} \leq 1\right) U(s)$, we find

$$
\frac{d}{d s} W_{0}(s)=i U_{0}(s)^{*}(A+B) U(s)
$$

where

$$
A=i(W(0, s)-W(x, s)) F\left(\frac{\langle x\rangle}{s^{\alpha}} \leq 1\right)
$$

and

$$
B=D F\left(\frac{\langle x\rangle}{s^{\alpha}} \leq 1\right) .
$$

By (2.3), $A=O\left(s^{\alpha-1-\mu}\right)$ and therefore contributes an integrable term into $\frac{d}{d s} W(s)$. Next, let $\delta<\frac{\alpha}{2}$. Using that

$$
D F\left(\frac{\langle x\rangle}{s^{\alpha}} \leq 1\right)=\frac{1}{s}\left(2 s^{1-\alpha} \gamma-\alpha \frac{\langle x\rangle}{s^{\alpha}}\right) F^{\prime}\left(\frac{\langle x\rangle}{s^{\alpha}} \leq 1\right)+O\left(s^{-2 \alpha}\right)
$$

and that $F^{\prime}\left(\langle x\rangle / s^{\alpha} \geq 1\right)=($ positive constant $) \cdot F\left(\langle x\rangle / s^{\alpha}=1\right)$, we find that due to Theorem 5.2

$$
F\left(s^{1-\alpha} \gamma \leq \delta\right) D F\left(\frac{\langle x\rangle}{s^{\alpha}} \leq 1\right) \doteq 0
$$


on $\operatorname{Ran} E_{\Omega}$. On the other hand, by Theorem 5.1 and $\sqrt{-\Delta}$-boundedness of $\gamma$ we have that

$$
F\left(s^{1-\alpha} \gamma \geq \delta\right) D F\left(\frac{\langle x\rangle}{s^{\alpha}} \leq 1\right)=O_{1 / 2}\left(s^{-2 \alpha-\eta+2}\right)
$$

on $\operatorname{Ran} F^{+}$. Thus $B$ also contributes an integrable term into $\frac{d}{d s} W(s)$. Collecting the last three statements and remembering (4.28) and Theorem 4.6 we conclude that

$$
\int_{0}^{t}\left|\frac{d}{d s}\left\langle W_{0}(s) \psi, u\right\rangle\right| d s \leq C\|\psi\|\|u\|
$$

for any $\psi \in \operatorname{Ran} F^{+}$and any $u \in L^{2}$, and with $C$ independent of the $\psi$ 's and $u$ 's. This inequality shows that $W_{0}(t)=W_{0}(1)+\int_{1}^{t} \frac{d}{d s} W_{0}(s) d s$ converges strongly as $t \rightarrow \infty$.

Theorem 6.1 implies that for any $g \in \operatorname{Ran}\left(F^{ \pm} Q^{ \pm}\right)$there is $\theta$ s.t.

$$
\left\|F\left(\frac{\langle x\rangle}{t^{\alpha}} \leq 1\right) g_{t}-U_{0}(t) \theta\right\| \rightarrow 0
$$

as $t \rightarrow+\infty$, provided $\mu>\alpha>\frac{3-\eta}{2}$. Combining this with (5.20), we obtain that, if $\mu>\frac{3-\eta}{2}$, then for any $g \in \operatorname{Ran}\left(F^{+} Q^{+}\right)$there is $\theta$ s.t.

$$
\left\|g_{t}-U_{0}(t) \theta\right\| \rightarrow 0
$$

as $t \rightarrow+\infty$.

Theorem 6.2. Let $\mu>\frac{3-\eta}{2}$. For any $\psi \in L^{2}$ and for any $\delta>0$ there are $a$ finite union, $\Omega$, of compact intervals disjoint from the threshold set of $H, \varepsilon>0$, and $T>0$ and there is $\theta$ s.t.

$$
\left\|\psi_{t}-F\left(\frac{\langle x\rangle}{t} \geq \varepsilon\right) \varphi_{t}-e^{-i H t} e^{-i \int_{0}^{t} W(0, s) d s} \theta\right\| \leq \delta
$$

for $t>T$. Here $\varphi$ is the projection of $\psi$ onto $\left(\operatorname{Ran} F^{+}\right)^{\perp}$.

Proof. We can decompose any $\psi \in L^{2}$ as $\psi=\varphi+g$ with

$$
\varphi \perp \operatorname{Ran} F^{+} \text {and } g \in \operatorname{Ran} F^{+} \text {. }
$$

We claim that for any $\delta>0$ there are $\varepsilon>0$ and $T \geq 0$ s.t.

$$
\left\|F\left(\frac{\langle x\rangle}{t} \leq \varepsilon\right) \varphi_{t}\right\| \leq \delta \text { for any } t \geq T .
$$

Indeed, denote $F_{\varepsilon}^{t}=U(t)^{*} F\left(\frac{\langle x\rangle}{t} \leq \varepsilon\right) U(t)$. Using that $F^{+} \varphi=0$, we obtain

$$
\left\|F\left(\frac{\langle x\rangle}{t} \leq \varepsilon\right) \varphi_{t}\right\| \leq\left\|\left(F_{\varepsilon}^{t}-F_{\varepsilon}^{+}\right) \varphi\right\|+\left\|\left(F_{\varepsilon}^{+}-F^{+}\right) \varphi\right\| .
$$

First for any $\delta>0$ we pick up $\varepsilon>0$ so that

$$
\left\|\left(F_{\varepsilon}^{+}-F^{+}\right) \varphi\right\| \leq \frac{\delta}{3}
$$


and then for $\delta>0$ and $\varepsilon>0$ we pick $T=T(\delta, \varepsilon)$ s.t.

$$
\left\|\left(F_{\varepsilon}^{t}-F_{\varepsilon}^{+}\right) \varphi\right\| \leq \frac{\delta}{3} \quad \text { for any } t \geq T(\delta, \varepsilon) .
$$

The last two relations yield (6.12).

Equations (6.9), (6.11), and (6.12) yield (6.10).

\section{ASYMPTOTIC CLUSTERING REVISITED}

In this section we show that under certain conditions the system in question breaks up into fragments propagating away from each other. We begin with definitions pertaining to such break ups. Denote by $a, b, \ldots$ partitions of the set $\{1, \ldots, N\}$ into nonempty disjoint subsets, called clusters. The relation $b<a$ means that $b$ is a refinement of $a$ and $b \neq a$. Then $a \vee b, a_{\min }$, and $a_{\max }$ are defined in a standard way. $|a|$ denotes the number of clusters in $a$. We also identify pairs $l=(i j)$ with partitions having $N-1$ clusters: $(i j) \leftrightarrow\{(i j)(1) \cdots(i) \cdots(j) \cdots(N)\}$. We emphasize that the relation $l \Varangle a$ (resp., $l \leq a$ ) with $l=(i j)$ is equivalent to saying that $i$ and $j$ belong to different clusters (resp. to same cluster) of $a$.

We define the intercluster interaction for a partition $a$ as $I_{a}=$ sum of all potentials linking different clusters in $a$, i.e., $I_{a}=\sum_{l \nless a} V_{l}$. For each $a$ we introduce the truncated Hamiltonian:

$$
H_{a}=H-I_{a} \text {. }
$$

This operator is clearly selfadjoint. It describes the motion of the original system broken into noninteracting clusters of particles.

For each cluster decomposition $a$, define the configuration space of relative motion of the clusters in $a$ :

$$
X_{a}=\left\{x \in X \mid x_{i}=x_{j} \text { if } i \text { and } j \text { belong to same cluster of } a\right\},
$$

and the configuration space of the internal motion within those clusters:

$$
X^{a}=\left\{x \in X \mid \sum_{j \in C_{i}} m_{j} x_{j}=0 \text { for all } C_{i} \in a\right\} .
$$

Clearly $X_{a}$ and $X^{a}$ are orthogonal (in our inner product) and they span $X$ :

$$
X=X^{a} \oplus X_{a} .
$$

Given a generic vector $x \in X$ (resp., $k \in X^{\prime}$ ), its projections on $X^{a}$ and $X_{a}$ (resp., $\left(X^{a}\right)^{\prime}$ and $X_{a}^{\prime}$ ) will be denoted by $x^{a}$ and $x_{a}$ (resp., $k^{a}$ and $k_{a}$ ). The momentum operators canonically conjugate to $x_{a}$ and $x^{a}$ and corresponding to $k_{a}$ and $k^{a}$ will be denoted by $p_{a}$ and $p^{a}$, respectively. Let $v_{a}=i\left[\left|p_{a}\right|^{2}, x_{a}\right]$, the cluster velocity operator.

For long-range potentials, even as clusters move away from each other, the intercluster interaction cannot be entirely ignored. Its effect remains in the form of time-dependent modification of the internal potentials of the clusters. 
We proceed to the definition of this modification. We introduce the following cut-off function:

$$
F_{a}(x)=\prod_{l \nsubseteq a} F\left[\left\langle x^{l}\right\rangle>1\right] .
$$

Note that this function is supported in the region of the configuration space in which the distance between clusters in $a$ is at least 1 . Now we are prepared to define the modified Hamiltonian of independent clusters:

$$
H_{a}(t)=H_{a}+W_{a}(t),
$$

where $W_{a}(t)$ is the family of pseudodifferential operators written in detail as

$$
W_{a}(t)=W_{a, t}\left(x^{a}, v_{a} t\right)
$$

(note here that $x^{a}$ and $v_{a}$ commute). Here (note the identification $x=$ $\left.\left(x^{a}, x_{a}\right)\right)$

$$
\begin{aligned}
& W_{a, t}(x)=W_{a}(x) F\left(\frac{\left\langle x_{a}\right\rangle}{|t|+1} \geq \lambda\right)+W(x, t), \\
& W_{a}(x)=I_{a}(x) F_{a}\left(\frac{x}{\delta\langle x\rangle}\right),
\end{aligned}
$$

where $\delta>0$ is a geometrical constant depending only on $N$ and the $m_{i}$ 's, and $\lambda>0$ is sufficiently small and is specified in appropriate places. Due to condition (B) the effective potentials $W_{a, t}$ obey the estimates

$$
\left|\partial_{x}^{\alpha} \partial_{t}^{\beta} W_{a, t}(x)\right| \leq C_{\alpha, \beta}(1+|x|+|t|)^{-\mu-|\alpha|-\beta}
$$

for $|\alpha|+|\beta| \leq 2$. These inequalities hold strictly speaking only for $t$ sufficiently large so that $\delta \lambda t>R$. However, in order not to complicate the notation we ignore this qualification.

Since $H_{a}$ is selfadjoint and $W_{a}(t)$ is uniformly (in $t$ ) bounded, one concludes that $H_{a}(t)$ generates the evolution $U_{a}(t)$ (see, e.g., [RSII]). More precisely, there is a family $U_{a}(t)$ of unitary operators (with a certain group property) which solves uniquely the Schrödinger Cauchy problem:

$$
i \frac{\partial U_{a}(t)}{\partial t}=H_{a}(t) U_{a}(t), \quad U_{a}(0)=\mathrm{id} .
$$

We introduce $U_{a, \lambda}(t)=F\left(\left|p_{a}\right| \geq \lambda\right) U_{a}(t)$, where $\lambda$ is the same as in (7.6). Of course $F\left(\left|p_{a}\right| \geq \lambda\right)$ commutes with $U_{a}(t)$. Observe also that $\left|v_{a}\right|=2\left|p_{a}\right|$, where the 1.h.s. norm is in $X$, while the r.h.s. is in $X^{\prime}$.

Theorem 7.1. Assume conditions (A) and (B) with $\mu>\frac{1}{2}$. Let $\phi$ be either $E_{\Delta}(1-P)$ with $\Delta$ a bounded interval disjoint from the thresholds of $H$ or $F\left(\frac{\langle x\rangle}{t} \geq \varepsilon\right) E_{\Delta}$ with $\varepsilon>0$ and $\Delta$ bounded. Then there are $\psi_{a}^{ \pm} \in L^{2}$ and $\lambda>0$ s.t.

$$
\left\|\phi \psi_{t}-\sum_{|a| \geq 2} U_{a, \lambda}(t) \psi_{a}^{ \pm}\right\| \rightarrow 0
$$


as $t \rightarrow \pm \infty$. Here $\lambda$ belongs to $\left(0, \delta \varepsilon_{1}\right)$, where $\delta>0$ depends on $N$, the $m_{i}$ 's, and $\Delta$ only, and where $\varepsilon_{1}=\min _{\lambda<E, \lambda \in \tau}(E-\lambda)^{1 / 2}$ with $E=\inf \Delta$ in the first case and $\varepsilon_{1}=\varepsilon$ in the second case.

When (7.9) holds we say that $\phi \psi_{t}$ is asymptotically clustering.

This theorem for $\mu=1$ is formulated in [SigSof6, Theorem 8.3] and its proof is essentially contained in [SigSof 1,3 ] (a simpler version is given in [Der1]) with minor adjustments discussed in [SigSof6]. To obtain the result for $\mu>\frac{1}{2}$ one combines the proof of the $\mu=1$ case with an observation of [GerDer]. The latter consists of proving the existence of the Deift-Simon wave operators in two steps, first, by comparing $U(t)$ with the evolution generated by $H_{a}+W_{a, t}(x)$ and then by comparing the latter evolution with $U_{a}(t)$ (generated by $H_{a}(t)=$ $\left.H_{a}+W_{a, t}\left(x^{a}, v_{a} t\right)\right)$. One can also derive Theorem 7.1 directly from the proof of [GerDer].

\section{ASYMPTOTIC COMPLETENESS}

In this section we combined results of $\S \S 5-7$ in order to prove asymptotic completeness. To begin with, we cast the intuitive notion of asymptotic completeness into precise mathematical terms. To this end we need the notion of channel. If $i$ and $j$ belong to some cluster of $a$, then $x_{i}-x_{j}=\left(\pi^{a} x\right)_{i}-\left(\pi^{a} x\right)_{j}$, where $\pi^{a}$ is the orthogonal projection in $X$ on $X^{a}$. This elementary fact and the fact that $-\Delta=\langle p, p\rangle$ with $p=-i \nabla_{x}$ (see equation (2.5) and the sentence after it) yield the following decomposition:

$$
H_{a}=H^{a} \otimes 1+1 \otimes T_{a} \text { on } L^{2}(X)=L^{2}\left(X^{a}\right) \otimes L^{2}\left(X_{a}\right) .
$$

Here $H^{a}$ is the Hamiltonian of the noninteracting $a$-clusters with their centersof-mass fixed at the origin, acting on $L^{2}\left(X^{a}\right)$, and $T_{a}=-\left(\right.$ Laplacian on $\left.X_{a}\right)$, the kinetic energy of the center-of-mass motion of those clusters.

The eigenvalues of $H^{a}$, whenever they exist, will be denoted by $\varepsilon^{\alpha}$, where $\alpha=(a, m)$ with $m$, the number of the eigenvalue in question counting the multiplicity. For $a=a_{\min }$, we set $\varepsilon^{\alpha}=0$. The set $\tau=\left\{\varepsilon^{\alpha}\right.$, all $\left.\alpha\right\}$ is called the threshold set of $H$ and $\varepsilon^{\alpha}$ are called the thresholds of $H$. For $\alpha=(a, m)$ we denote $|\alpha|=|a|$ and $a(\alpha)=a$.

Pairs $\alpha=(a, m)$ are called the channels. Each pair can be identified with an asymptotic state of the system in question as $t \rightarrow+\infty$ or $t \rightarrow-\infty: a$ specifies a decomposition of the system into subsystems and $m$ specifies a stable motion (i.e., a bound state) within each subsystem of this decomposition. Next we define the channel Hamiltonians for the long-range scattering as

$$
H_{\alpha}(t)=\varepsilon^{\alpha}+\left|p_{a}\right|^{2}+W_{a}\left(0, v_{a} t\right)
$$

on $L^{2}\left(X_{a}\right)$. Denote now by $U_{\alpha}(t)$ the evolution on $L^{2}\left(X_{a}\right)$ generated by $H_{\alpha}(t)$ :

$$
i \frac{\partial}{\partial t} U_{\alpha}(t)=H_{\alpha}(t) U_{\alpha}(t) \text { and } U_{\alpha}(0)=\mathrm{id} .
$$

$U_{\alpha}(t)$ are the channel evolution operators. We now can formulate the property of asymptotic completeness. We say that a system obeying (B) (see $\S 2$ ) with 
$\mu>\frac{1}{2}$ is asymptotically complete if for any $\psi \in L^{2}(X)$

$$
\left\|\psi_{t}-\sum_{\alpha} \psi^{\alpha} \otimes U_{\alpha}(t) u_{\alpha}^{ \pm}\right\| \rightarrow 0
$$

as $t \rightarrow \pm \infty$ for some $u_{\alpha}^{ \pm}$. Here $\psi^{\alpha}$ are the eigenfunctions of $H^{a}$ corresponding to eigenvalues $\varepsilon^{\alpha}$ and the sum extends over all channels including a trivial one with $|a(\alpha)|=1$.

This definition is equivalent to a standard one in terms of the channel wave operators (provided the latter exist). The smoothness restriction in condition (B) is immaterial, it can be replaced by any other suitable condition. However, the restriction on the decay is essential (see [Sig1]).

We formulate the main result of this paper.

Theorem 8.1. Assume conditions (A)-(B) hold with $\mu>\frac{3-\eta}{2}$, where $\eta=$ $\left(1-2^{-N-1}\right)^{-1}$. Then asymptotic completeness holds.

Proof. First of all Theorem 6.2 states that for any $\psi \in L^{2}$ and for any $\delta>0$ there are $\varepsilon>0, T>0$, and $\varphi, \theta \in L^{2}$ s.t.

$$
\left\|\psi_{t}-F\left(\frac{\langle x\rangle}{t} \geq \varepsilon\right) \varphi_{t}-e^{-i H t} e^{-i \int_{0}^{t} W(0, s) d s} \theta\right\| \leq \delta
$$

for $t \geq T$. For $\delta>0$ we pick up a neighbourhood, $\Omega^{\prime}$, of $\tau \cup\{\infty\}$, where, recall, $\tau$ is the threshold set of $H$, so that

$$
\left\|E_{\Omega^{\prime}} \theta\right\| \leq \delta \text {. }
$$

Let $\Omega$ be a compact set disjoint from the thresholds of $H$ and s.t.

$$
E_{\Omega}+E_{\Omega^{\prime}}=1 \text {. }
$$

Then, by Theorem 7.1, $E_{\Omega} \bar{P} e^{-i H t} \theta$, where $\bar{P}=1-P$, is asymptotically clustering, i.e., there are $\lambda>0$ and $\theta_{a}$ s.t.

$$
\left\|e^{-i H t} e^{-i \int_{0}^{t} W(0, s) d s} E_{\Omega} \bar{P} \theta-\sum_{|a| \geq 2} U_{a, \lambda}(t) \theta_{a}\right\| \rightarrow 0
$$

as $t \rightarrow+\infty$. Here $U_{a, \lambda}(t)$ are the evolution operators described in $\S 7$ (after (7.8)). For the discrete part we have

$$
e^{i H t} e^{-i \int_{0}^{t} W(0, s)} E_{\Omega} P \theta=\sum e^{-i\left(\varepsilon^{\alpha} t+\int_{0}^{t} W(0, s) d s\right)} P_{\alpha} E_{\Omega} \theta,
$$

where the sum is taken over all $\alpha$ 's s.t. $|a(\alpha)|=1$ and $\varepsilon^{\alpha} \in \Omega$. Since $\Omega$ is disjoint from the thresholds of $H$, the sum on the r.h.s. is finite.

By [SigSof4, Theorem 4.1(ii)] for any $\delta>0$ there is a bounded set $\Omega_{1}$ s.t.

$$
\sup _{t}\left\|\left(1-E_{\Omega_{1}}\right) \varphi_{t}\right\| \leq \delta \text {. }
$$

Next, by Theorem 7.1, $F\left(\frac{\langle x\rangle}{t} \geq \varepsilon\right) E_{\Omega_{1}} \varphi_{t}$ is asymptotically clustering, i.e., there are $\lambda>0$ and $\varphi_{a} \in L^{2}$ s.t.

$$
\left\|F\left(\frac{\langle x\rangle}{t} \geq \varepsilon\right) E_{\Omega_{1}} \varphi_{t}-\sum_{|a| \geq 2} U_{a, \lambda}(t) \varphi_{a}\right\| \rightarrow 0
$$


as $t \rightarrow+\infty$. Combining equations (8.4) $-(8.10)$ yields that for any $\delta>0$ there are $\lambda>0$, a finite subset, $\mathscr{A}$, of the $\alpha$ 's with $|a(\alpha)|=1, \psi_{a} \in L^{2}$, and $\theta \in L^{2}$ s.t.

$$
\sup _{t}\left\|\psi_{t}-\sum_{\alpha \in \mathscr{A}} U_{\alpha}(t) P_{\alpha} \theta-\sum_{|a| \geq 2} U_{a, \lambda}(t) \psi_{a}\right\| \leq \delta .
$$

Applying this statement inductively and taking into account that $P_{\alpha} U_{a, \lambda}(t)=$ $U_{\alpha}(t) P_{\alpha} F\left(\left|p_{a}\right| \geq \lambda\right)$ for $a(\alpha)=a$, we conclude that for any $\psi \in L^{2}$ and $\delta>0$ we can find $\varphi_{\alpha, \delta} \in L^{2}$ and a finite subset, $\mathscr{B}$, of the $\alpha$ 's s.t.

$$
\sup _{t}\left\|\psi_{t}-\sum_{\alpha \in \mathscr{B}} U_{\alpha}(t) P_{\alpha} \varphi_{\alpha, \beta}\right\| \leq \delta .
$$

On the other hand, by a standard argument the wave operators

$$
\operatorname{s-lim} U(t)^{*} P_{\alpha} U_{\alpha}(t)
$$

exist. The last two results yield (8.3).

\section{APPENDIX: OPERATOR $\gamma_{t}$}

The analysis of this supplement is motivated by [SigSof 1, 3] and, to a larger extent, by [Gr]. Some of the analysis of $\S 7$ simplifies if we use the operator $\gamma_{t}$, constructed in this supplement, instead of $\gamma$. An important fact is that though $\gamma_{t}$ is close to $\gamma$, its Heisenberg derivative has a useful lower bound globally. The main result of this section is

Theorem A.1. There is a family, $\gamma_{t}$, of selfadjoint operators s.t. $(-\Delta+1)^{-s} \gamma_{t}(-\Delta+1)^{-1 / 2+s}$ is bounded and norm differentiable for any $s \in\left[0, \frac{1}{2}\right]$ and s.t.

$$
\begin{gathered}
\gamma_{t}=\gamma+O\left(t^{-\eta_{1}}\right), \\
{\left[\gamma_{t},\left[\gamma_{t}, H\right]\right]=O_{1}\left(t^{-\eta_{2}}\right),}
\end{gathered}
$$

and

$$
D \gamma_{t} \geq-C t^{-\eta}
$$

with $\eta_{1}>\frac{\eta-1}{2}>0$ and $\eta_{2}>\eta>1$, depending entirely on $N$ and $\mu$, and with the constant independent of $t$. If $\mu \geq \frac{2}{3}$, we can choose then $\eta_{1}=2^{-N-1}, \eta_{2}=$ $\left(1-2^{-N}\right)^{-1}$, and $\eta=\left(1-2^{-N-1}\right)^{-1}$.

Below we construct $\gamma_{t}$ explicitly and prove an estimate stronger than (A.3) and which implies positivity of $D \gamma_{t}$ sandwiched by appropriate energy cut-offs. Before proceeding to the proof we introduce some definitions and notation. We introduce selfadjoint operators

$$
\gamma^{a}=\frac{1}{2}\left(\left\langle p^{a}, \hat{x}^{a}\right\rangle+\left\langle\hat{x}^{a}, p^{a}\right\rangle\right)
$$


and

$$
\gamma_{a}=\frac{1}{2}\left(\left\langle p_{a}, \hat{x}_{a}\right\rangle+\left\langle\hat{x}_{a}, p_{a}\right\rangle\right)
$$

related to the internal motion of the clusters and to the motion of the centersof-mass of the clusters.

Let numbers $\delta_{a} \in\left(\max \left(\frac{1}{2}, \frac{1}{1+\mu}\right), 1\right)$ satisfy $\delta_{b}<\delta_{a}$ if $b<a$. Moreover, we assume that $2 \min _{b>a} \delta_{b}>\delta_{a}+1$ for any $a$ with $|a| \geq 2$. Let $t \geq 1$ and $t_{a}=t^{\delta_{a}}$. [SigSof6] have constructed the following partition of unity (see also [SigSof1] and, especially, [Gr]): $0 \leq j_{a, t}(x) \leq 1, j_{a, t}(x)$ are smooth in $x$ and $t$ and obey

$$
\begin{gathered}
\sum j_{a, t}(x)^{2}=1, \\
\operatorname{supp} j_{a, t} \subset\left\{\left|x^{a}\right| \leq t_{a},|x|_{a} \geq c \bar{t}_{a}\right\}
\end{gathered}
$$

with some geometrical constant $c$ dependent only on $N$ and the $m_{i}$ 's and with

$$
\begin{gathered}
\bar{t}_{a}=\min _{b>a} t_{b}, \\
\partial_{x}^{\beta} j_{a, t}(x)=O\left(t_{a}^{-|\beta|}\right),
\end{gathered}
$$

for any $\beta$, and

$$
\nabla_{x} j_{a, t}=-\frac{\hat{x}^{a}}{t_{a}} \chi_{a}+O\left(\bar{t}_{a}^{-1}\right),
$$

where $0 \leq \chi_{a} \leq 1$ is a smooth function supported in $\operatorname{supp} j_{a, t}$ and obeying $\partial^{\beta} \chi_{a}=O\left(t_{a}^{-|\beta|}\right)$. Moreover,

$$
\partial_{t} j_{a, t}=O\left(t^{-1}\right)
$$

Thus $\left\{j_{a, t}\right\}$ is a smooth partition of unity describing various break-ups of the system.

Remarks A.2. (a) [SigSof6] defines $j_{a, t}$ for $|a| \geq 2$ satisfying $\sum_{|a| \geq 2} j_{a, t}=$ $F\left(\frac{\langle x\rangle}{t_{a_{\max }}} \geq 1\right)$. Thus adding to this collection $j_{a, t}=F\left(\frac{\langle x\rangle}{t_{a}} \leq 1\right)$ for $a=a_{\max }$ and renormalizing produces the desired partition of unity. (b) In fact, the partition in [SigSof6] is defined for arbitrary scales $t_{a}$ satisfying $t_{b}<(10 M)^{-1} t_{a}$ if $b<a$ with $M$ given by $M=\max _{a, b} \sup _{x}\left(\left|x^{a \vee b}\right|\left(\left|x^{a}\right|+\left|x^{b}\right|\right)^{-1}\right)<\infty$.

Equations (A.8), (A.9), and (A.10) and a simple computation show that

$$
D j_{a, t}=-2 t_{a}^{-1} \gamma^{a} \chi_{a}+O_{1}\left(\bar{t}_{a}^{-1}\right)+O\left(t_{a}^{-2}\right)
$$

Now we define

$$
\gamma_{t}=\sum j_{a, t} \gamma_{a} j_{a, t} .
$$

Proof of Theorem A.1. Using that by (A.8)

$$
\left[\gamma_{a}, j_{a, t}\right]=-i \hat{x}_{a}\left(\nabla_{a} j_{a, t}\right)=O\left(t_{a}^{-1}\right),
$$


we obtain

$$
\gamma_{t}=\sum\left(j_{a, t}\right)^{2} \gamma_{a}+O\left(\max t_{a}^{-1}\right)
$$

This together with (A.7) yields

$$
\gamma_{t}=\gamma+R+O\left(\max t_{a}^{-1}\right)
$$

where

$$
R=\sum\left(j_{a, t}\right)^{2}\left(\gamma_{a}-\gamma\right)
$$

It remains to estimate $R$. Ignoring higher order terms, we assume $\hat{x}=x\langle x\rangle^{-1}$, etc. Using that

$$
\begin{aligned}
\hat{x}_{a} \cdot p_{a} & =\left\langle x_{a}\right\rangle^{-1} x_{a} \cdot p_{a}=\left\langle x_{a}\right\rangle^{-1}\left(x \cdot p-x^{a} \cdot p^{a}\right) \\
& =\frac{\langle x\rangle}{\left\langle x_{a}\right\rangle} \hat{x} \cdot p-\frac{\left\langle x^{a}\right\rangle}{\left\langle x_{a}\right\rangle} \hat{x}^{a} \cdot p^{a}
\end{aligned}
$$

we find

$$
\gamma_{a}-\gamma=\left(\frac{\langle x\rangle}{\left\langle x_{a}\right\rangle}-1\right) \gamma-\frac{\left\langle x^{a}\right\rangle}{\left\langle x_{a}\right\rangle} \gamma^{a}+O\left(\left\langle x_{a}\right\rangle^{-1}\right) .
$$

Next, using that

$$
\langle x\rangle\left\langle x_{a}\right\rangle^{-1}=\frac{\langle x\rangle-\left\langle x_{a}\right\rangle}{\left\langle x_{a}\right\rangle}=\frac{\left\langle x^{a}\right\rangle^{2}}{\left(\langle x\rangle+\left\langle x_{a}\right\rangle\right)\left\langle x_{a}\right\rangle}+O\left(\left\langle x_{a}\right\rangle^{-2}\right),
$$

we obtain

$$
\gamma_{a}-\gamma=\frac{\left\langle x^{a}\right\rangle^{2}}{\left(\langle x\rangle+\left\langle x_{a}\right\rangle\right)\left\langle x_{a}\right\rangle} \gamma-\frac{\left\langle x^{a}\right\rangle}{\left\langle x_{a}\right\rangle} \gamma^{a}+O\left(\left\langle x_{a}\right\rangle^{-1}\right) .
$$

Using this relation, the definition of $R,\left(\right.$ A.6), and that $\gamma$ and $\gamma^{a}$ are $\sqrt{-\Delta}$ bounded, we obtain

$$
\begin{aligned}
R & =O_{1 / 2}\left(\max _{a} t_{a}^{2} \cdot \bar{t}_{a}^{-2}\right)+O_{1 / 2}\left(\max _{a} t_{a} \cdot \bar{t}_{a}^{-1}\right)+O\left(\max _{a} \bar{t}_{a}^{-1}\right) \\
& =O_{1 / 2}\left(\max _{a}\left(t_{a} \cdot \bar{t}_{a}^{-1}\right) .\right.
\end{aligned}
$$

This together with (A.14) and the relations $t_{a}=t^{\delta_{a}}$ and $\bar{t}_{a}=\min _{b>a} t_{b}$ yields (A.1) with $\eta_{1}=\min _{a}\left(\min _{b>a} \delta_{b}-\delta_{a}\right)>0$.

(A.2) with $\eta_{2}=2 \min _{a} \delta_{a}>1$ results from a straightforward computation. Now we prove (A.3). Using that

$$
i\left[H, \gamma_{a}\right]=4 \frac{1}{\sqrt{\left\langle x_{a}\right\rangle}}\left(p_{a}^{2}-\gamma_{a}^{2}\right) \frac{1}{\sqrt{\left\langle x_{a}\right\rangle}}+O\left(\left\langle x_{a}\right\rangle^{-2}\right)+O\left(\nabla_{a} I_{a}\right)
$$

and that $\nabla I_{a}=O\left(\left(|x|_{a}\right)^{-1-\mu}\right)$ and (A.6), we find

$$
\begin{aligned}
D \gamma_{t}= & 2 \sum j_{a, t} \frac{1}{\sqrt{\left\langle x_{a}\right\rangle}}\left(p_{a}^{2}-\gamma_{a}^{2}\right) \frac{1}{\sqrt{\left\langle x_{a}\right\rangle}} j_{a, t} \\
& +R_{1}+O\left(\max _{a} t_{a}^{-1-\mu}\right),
\end{aligned}
$$


where we have assumed that $\mu \leq 1$ and where

$$
R_{1}=4 \sum\left(D j_{a, t}\right) \gamma_{a} j_{a, t}+\text { adjoint }
$$

Using (A.19) and the relation $\sum D\left(j_{a, t}^{2}\right)=0$, and using (A.6), we get

$$
\begin{aligned}
R_{1}= & -\sum D j_{a, t} \frac{\left\langle x^{a}\right\rangle}{\left\langle x_{a}\right\rangle} \gamma^{a} j_{a, t} \\
& +O_{1 / 2}\left(\max t_{a}^{-2}\right)+O_{1}\left(\max t_{a}^{-1} \cdot t_{a}^{2} \cdot \bar{t}_{a}^{-2}\right)+O_{1 / 2}\left(\max t_{a}^{-1} \cdot \bar{t}_{a}^{-1}\right) .
\end{aligned}
$$

Now remembering (A.11), we find

$$
R_{1}=2 \sum t_{a}^{-1} \gamma^{a} \chi_{a} \frac{\left\langle x^{a}\right\rangle}{\left\langle x_{a}\right\rangle} j_{a, t} \gamma^{a}+O_{1 / 2}\left(\max t_{a}^{-2}\right)+O_{1}\left(\max t_{a} \cdot \bar{t}_{a}^{-2}\right)
$$

Combining (A.20) and (A.21), we obtain

$$
\begin{aligned}
D \gamma_{t}= & 2 \sum j_{a, t} \frac{1}{\sqrt{\left\langle x_{a}\right\rangle}}\left(p_{a}^{2}-\gamma_{a}^{2}\right) \frac{1}{\sqrt{\left\langle x_{a}\right\rangle}} j_{a, t}+2 \sum \gamma^{a} \frac{\chi_{a}\left\langle x^{a}\right\rangle}{t_{a}\left\langle x_{a}\right\rangle} \gamma^{a} \\
& +O_{1 / 2}\left(\max t_{a}^{-2}\right)+O_{1}\left(\max t_{a} \cdot \bar{t}_{a}^{-2}\right)+O\left(\max t_{a}^{-1-\mu}\right) .
\end{aligned}
$$

Since each of the first two terms on the r.h.s. is positive $\left(p_{a}^{2} \geq \gamma_{a}^{2}\right)$, this yields (A.3) with $\eta=\min _{a} \min \left(2 \min _{b>a} \delta_{b}-\delta_{a},(1+\mu) \delta_{a}\right)$. By our choice of the $\delta_{a}$ 's, $\eta>1$ and $\eta_{2}>\eta$.

Now take $\delta_{a}=\delta_{|a|}$ with $(1+\mu)^{-1}<\delta_{N}<\cdots<\delta_{1}<1$. Pick up $\delta_{j}=$ $2^{j-1} \delta_{1}-\left(2^{j-1}-1\right) b$ and $b=\delta_{1}\left[1-\frac{\mu}{1+\mu} 2^{1-N}\right]^{-1}$. Taking $\delta_{1}$ very close to 1 and observing that, if $\mu>\frac{2}{3}$, then $b>\left(1-2^{-N-1}\right)^{-1}$, we conclude that

$$
\eta_{1}>2^{-N-1}, \quad \eta_{2}>\left(1-2^{-N}\right)^{-1}, \quad \text { and } \eta>\left(1-2^{-N-1}\right)^{-1} \text {. }
$$

Thus (A.1)-(A.3) are obeyed for our choice of the $\eta$ 's.

Note here that equation (A.22) and the first part of Lemma 3.1 imply Theorem A.2. Let $j_{a, t}$ be as above. Then

$$
\int_{1}^{\infty}\left\|\left(p_{a}^{2}-\gamma_{a}^{2}\right)^{1 / 2} \frac{1}{\sqrt{\left\langle x_{a}\right\rangle}} j_{a, t}(H+i)^{-1} \psi_{t}\right\|^{2} \frac{d t}{t} \leq C\|\psi\|^{2}
$$

for any $\psi \in L^{2}$ and with $C$ independent of the $\psi$ 's.

Observe that $p_{a}^{2}-\gamma_{a}^{2} \geq 0$ and

$$
p_{a}^{2}-\gamma_{a}^{2}=p_{a} \cdot\left[p_{a}-\frac{1}{2}\left(\gamma_{a} \hat{x}_{a}+\hat{x}_{a} \gamma_{a}\right)\right]+O_{1 / 2}\left(\left\langle x_{a}\right\rangle^{-1}\right)
$$

Hence (A.23) contains a rather strong statement about propagation: $\psi_{t}$ "vanishes" on $\left\{k_{a} /\left|k_{a}\right|=x_{a} /\left|x_{a}\right|\right\} \cap \operatorname{supp} j_{a, t}$. It can be used as one of the key ingredients in the proof of asymptotic clustering (Theorem 7.1). This approach is similar to that of [Gr]. 


\section{ACKNOWLEDGMENT}

It is a pleasure to thank J. Derezinski for communicating his ideas on asymptotic completeness. The material of the Appendix was worked out while the first author was visiting ETH-Zürich in the fall of 1989, while the rest of the proof in this paper was done during the first author's stay in Aarhus and the second author's stay at ETH-Zürich in July of 1991. The authors are grateful to J. Fröhlich and W. Hunziker, and to E. Balslev and A. Jensen for their hospitality.

\section{REFERENCES}

[CFKS] H. Cycon, R. Froese, W. Kirsch, and B. Simon, Schrödinger operators, Springer-Verlag, Berlin, Heidelberg, and New York, 1987.

[Der1] J. Derezinski, A new proof of the propagation theorem for $N$-body quantum systems, Comm. Math. Phys. 122 (1989), 203-231.

[Der2] _ Algebraic approach to the N-body long-range scattering, Rev. Math. Phys. 3 (1991), $1-62$.

[Der3] _ Private communication, July 1991.

[En1] V. Enss, Asymptotic observables on scattering states, Comm. Math. Phys. 89 (1983), 245268.

[En2] _ Quantum scattering theory for two- and three-body systems with potentials of shortand long-range, Schrödinger Operators (S. Graffi, ed.), Lecture Notes in Math., vol. 1159, Springer-Verlag, Berlin and New York, 1985.

[En3] _ Two-and three-body quantum scattering: completeness revisited, Symposium: "Partial Differential Equations" (Holzhou, 1988) (M. Demuth and B.-W. Schultze, eds.), Teubner-Texte Math., Teubner, Leipzig, 1989, pp. 108-120.

[GerDer] C. Gérard and J. Derezinski, A remark on asymptotic clustering for $N$-particle quantum systems, preprint, École Polytech., Paris, 1991.

[Gr] G.-M. Graf, Asymptotic completeness for $N$-body short-range quantum systems: a new proof, Comm. Math. Phys. 132 (1990), 73-101.

[Mo] E. Mourre, Opérateurs conjugués et propriétés de propagation, Comm. Math. Phys. 91 (1983), 279-300.

[RS] M. Reed and B. Simon, Methods of modern mathematical physics. II, Academic Press, San Diego, CA, 1979.

[Sig1] I. M. Sigal, Mathematical questions of quantum many-body problem, Seminaire sur les équations aux derivées partielles, 1986-1987, Exposé No. XXIII, École Polytech., Palaiseau, 1987.

[Sig2] _ On the long-rang scattering, Duke Math. J. 60 (1990), no. 2.

[SigSof1] I. M. Sigal and A. Soffer, The $N$-particle scattering problem: asymptotic completeness for short-range systems, Anal. Math. 126 (1987), 35-108.

[SigSof2] —, Asymptotic completeness of multiparticle scattering, Differential Equations and Mathematical Physics (Knowles and Saito, eds.), Lecture Notes in Math., vol. 1285, Springer-Verlag, Berlin, Heidelberg, and New York, 1987.

[SigSof3] __, Long-range many-body scattering. Asymptotic clustering for Coulomb-type potential, Invent. Math. 99 (1990), 115-143.

[SigSof4] _ Local decay and velocity bounds, preprint.

[SigSof5] _ _ Asymptotic completeness for Coulomb-type 3-body systems, preprint, Princeton, 1991. 
[SigSof6] _ Asymptotic completeness for $N \leq 4$ particle systems with the Coulomb-type interactions, Duke Math. J. 71 (1993), 243-298.

[SS] A. G. Sigalov and I. M. Sigal, Description of the spectrum of the energy operator of quantum mechanical systems invariant under permutations of identical particles, Theoret. and Math. Phys. 5 (1970), 990-1005.

ABSTRACT. We prove asymptotic completeness for $N$-particle long-range system with potentials vanishing as $O\left(|x|^{-\mu}\right)$, where $\mu \geq 1-2^{-N-2}$, at infinity.

Department of Mathematics, University of Toronto, Toronto, Ontario, Canada M5S $1 \mathrm{~A} 1$

E-mail address: ida@math.toronto.edu

Department of Mathematics, Princeton University, Princeton, New Jersey 08544

E-mail address: avy@math.princeton.edu 